\title{
A Novel System for Wind Speed Forecasting Based on Multi-Objective Optimization and Echo State Network
}

\author{
Jianzhou Wang, Chunying Wu * and Tong Niu \\ School of Statistics, Dongbei University of Finance and Economics, Dalian 116025, China; \\ wangjz@dufe.edu.cn (J.W.); Tong.Niu@uts.edu.au (T.N.) \\ * Correspondence: 201712288@mail.sdu.edu.cn; Tel.: +86-188-4265-2596
}

Received: 14 December 2018; Accepted: 17 January 2019; Published: 19 January 2019

\begin{abstract}
Given the rapid development and wide application of wind energy, reliable and stable wind speed forecasting is of great significance in keeping the stability and security of wind power systems. However, accurate wind speed forecasting remains a great challenge due to its inherent randomness and intermittency. Most previous researches merely devote to improving the forecasting accuracy or stability while ignoring the equal significance of improving the two aspects in application. Therefore, this paper proposes a novel hybrid forecasting system containing the modules of a modified data preprocessing, multi-objective optimization, forecasting, and evaluation to achieve the wind speed forecasting with high precision and stability. The modified data preprocessing method can obtain a smoother input by decomposing and reconstructing the original wind speed series in the module of data preprocessing. Further, echo state network optimized by a multi-objective optimization algorithm is developed as a predictor in the forecasting module. Finally, eight datasets with different features are used to validate the performance of the proposed system using the evaluation module. The mean absolute percentage errors of the proposed system are $3.1490 \%, 3.0051 \%$, $3.0618 \%$, and $2.6180 \%$ in spring, summer, autumn, and winter, respectively. Moreover, the interval prediction is complemented to quantitatively characterize the uncertainty as developing intervals, and the mean average width is below 0.2 at the $95 \%$ confidence level. The results demonstrate the proposed forecasting system outperforms other comparative models considered from the forecasting accuracy and stability, which has great potential in the application of wind power systems.
\end{abstract}

Keywords: wind speed forecasting; echo state network; forecasting accuracy; stability and practicality; hybrid forecasting system; interval prediction

\section{Introduction}

Advances in the development and utilization of renewable energies have greatly decreased environmental pollution and brought about extensive socio-economic benefits. Among various renewable energies, wind energy, as a kind of emission-free, cheap, inexhaustible, and accessible energy, is of great significance in the world energy structure. From the Renewables 2018 Global Status Report (GSR) [1], until the end of 2017, the global wind turbines' installed capacity achieved about a 539 GW increase by nearly $11 \%$, with more than 52 GW newly added in 2017, and the installed capacity got its third largest growth year ever. For many countries, wind power has become the backbone of strategies to phase out fossil and nuclear energy. In 2017, 43\% of Denmark's power came from wind, setting a new world record. A growing number of countries have reached a double-digit share of wind power, including Germany, Spain, Sweden, Portugal, Ireland, and Uruguay. Until the end of 2017, China was still the largest wind power market with a capacity of $190 \mathrm{GW}$ installed and will continue its undisputed global leader position of wind power. Figure 1 shows the distribution of global leading countries in wind power installation capacities in 2017. Wind power has become one of the 
fastest increasing renewable energies around the world and greatly impacts the sustained economic development $[2,3]$.

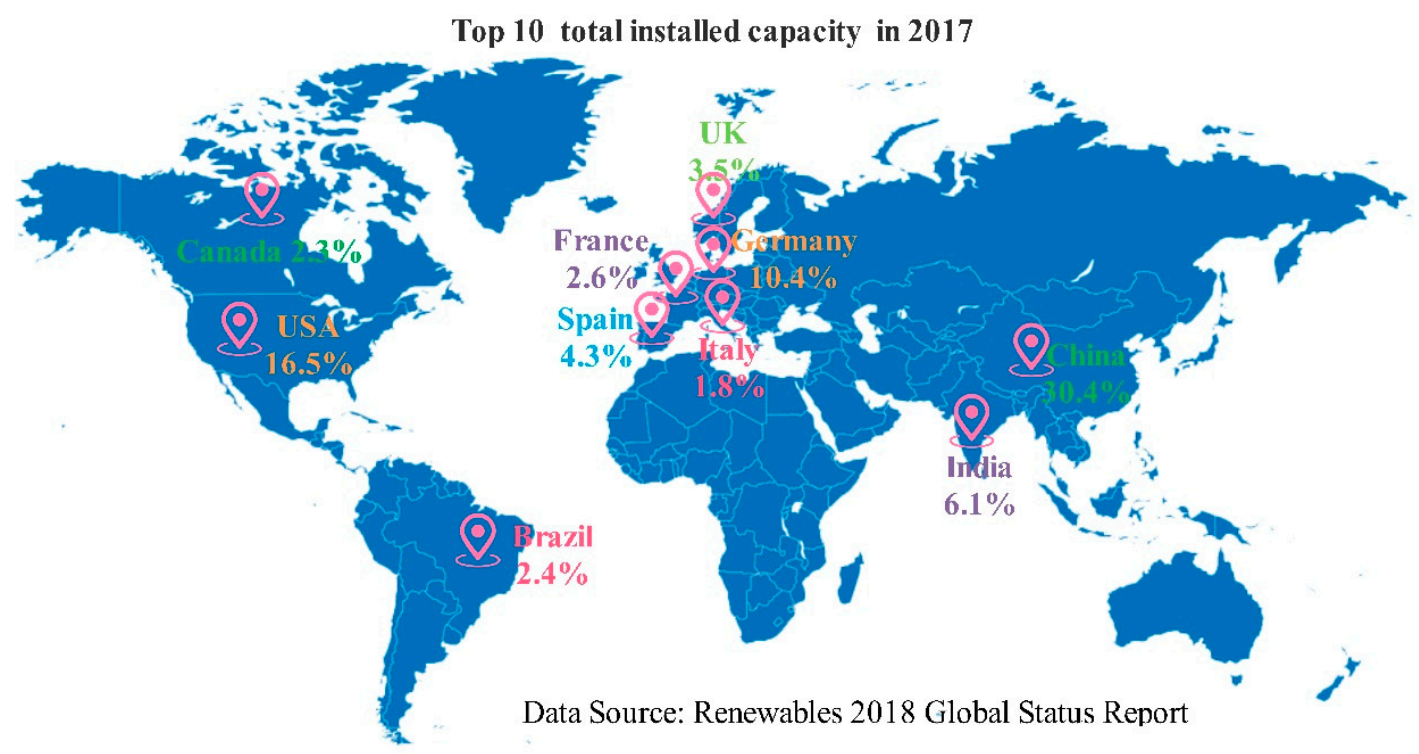

Figure 1. The distribution of the global wind power capacity in 2017.

However, the power integration faces a considerable challenge because of the inherent highly stochastic and intermittent nature of wind power, which unavoidably impairs not only the dispatch and management of the electric system but also the stability of the power grid [4]. Therefore, wind speed evaluation as well as wind speed forecasting (WSF) is an effective way to address the above problems and to accomplish secure and reliable electricity dispatch and management. Accurate and stable WSF helps the power system satisfy the electricity demands of economic and social development on the basis of being resource-conserving and environment-friendly [5,6]. Considering the significance, researchers have carried on a large amount of research about both the theory and practice of WSF. Furthermore, to achieve better predictions, more and more approaches are developed for WSF that could be generally grouped to four classes [7]: physical models, statistical models, artificial intelligence models, as well as hybrid models.

Physical models, based on numerical weather prediction (NWP), generally employ meteorological data and geographic feature information to forecast future wind speeds [8-10]. With the development of numerical simulation, reanalysis data $[11,12]$ and satellite data $[13,14]$ have been used in wind speed evaluation gradually. They usually require much more for the model parameters so that the application of physical methods is inevitably restricted by the uncertain weather conditions and the performance of NWP. In the situation of climate change, researches use climate models $[15,16]$ to predict the future wind speed. Giménez, P.O. [17] demonstrated that reanalysis data and satellite data facilitate a reliable wind speed evaluation; they also considered the impacts of climate change on wind speed. Considering the various data types and parameters, the physical methods can achieve high forecasting accuracy. However, they bring on considerable computation time and computation complexity. Furthermore, they are unsatisfactory for short-term wind speed forecasting (STWSF).

Statistical models are much more appropriate for STWSF, which make the utmost of the historical data to model their inner relationship and then to build statistical models to predict the future wind speeds [18]. These methods have better forecasting performance and faster processing speeds. However, these models are based on the linear assumption so that they cannot describe exactly the wind speed time series which is essentially non-linear.

Recently, with the rapid development and extension of artificial intelligence techniques, researches have been aware of the artificial intelligence's excellent ability to deal with the nonlinear feature of wind speed data [19]. Artificial intelligence, for instance, the artificial neural network (ANN), the 
support vector machine (SVM), as well as the extreme learning machine (ELM), has been resoundingly employed for wind speed forecasting. An artificial intelligence technique is equipped to handle complex relationships and to make decisions under uncertainty based on its excellent self-learning and self-adaptation ability [20]. However, the common ANN like back propagation neural network (BPNN) that demands intricate training processing [21] may be easily trapped into local optimum [19] and needs a long training time. Other artificial intelligence techniques like SVM are of a compute burden associated with the sample size [22-27]. Therefore, it is hard to achieve wonderful wind speed forecasting performances using these methods. Echo state network (ESN), which has shown successful applications to time series forecasting like load forecasting, stock forecasting, energy consumption forecasting, and so on, can deal with the difficulties mentioned above but is still rarely applied in wind speed time series forecasting [28-32]. According to the literature [33-37], ESN replaces the hidden layer with a reservoir composed of many interconnected neurons so that it can (a) Reduce computation complexity and improving training efficiency; (b) achieve a higher forecasting accuracy; (c) capture complex dynamics in nonlinear time series; and (d) help avoid over-fitting problem. Therefore, ESN is competent for wind speed forecasting and can show an outstanding ability in practical applications.

Obviously, the application of ESN will make great progress in wind power forecasting, but the inherent stochastic and intermittent nature of wind power works against getting satisfactory forecasting results. Thus, to overcome the existing problems and to achieve a high accuracy of wind speed forecasting, researchers try to combine multifarious algorithms based on their individual advantages called hybrid methods. Commonly, a hybrid model exerts a certain mechanism to preprocess the original signals and then predicts through an optimized predictor. Zhang et al. [38] proposed a hybrid system which compounds hybrid backtracking search algorithm (HBSA), optimized variational mode decomposition (OVMD), as well as ELM. Results obtained from the research indicate the more satisfying performance in wind speed forecasting of hybrid models. Likewise, Du et al. [39] applied complementary ensemble empirical mode decomposition (CEEMD) and Elman neural network constituting a new hybrid model, which achieved more accurate results in wind speed forecasting. Researches demonstrate that hybrid models are capable of improving the precision effectively in wind speed forecasting.

Based on the literature research, the defect of the aforementioned methods can be summarized as (1) physical models, including reanalysis data, satellite data, and climate models, are unsatisfactory for STWSF and the computation time and computation complexity are considerable as well; (2) statistical models cannot describe exactly the essentially non-linear and fluctuation features of wind speed, but they are effective in predicting under small change of climate and other influencing factors, though the prediction performance is not ideal in the case of a large variety of random factors or poor-quality data; (3) artificial intelligence methods can capture the essentially nonlinearity and improve the accuracy of STWSF, but they also have some disadvantages, such as easy to be trapped into local optimum, over-fitting, and so on; and (4) hybrid models combine data preprocessing, optimization, and artificial intelligence methods to overcome the drawbacks and achieve better forecasting performance. Researches indicates that hybrid models can capture the feature of wind speed and combine the advantage of different methods, having great significance in improving the accuracy of STWSF [39].

Researchers have made a lot of improvement in wind speed forecasting models, but most researches focus on point forecasting and ignore the uncertainty in the forecasting process, which will pose risks to the operation and management of the power system. To analyze the uncertainty, Li et al. [6] conducted an interval prediction using a hybrid model to gain both high coverage and small width. Naik et al. [40] proposed a hybrid model based on the low rank Multi-kernel ridge regression and obtained a good performance in the wind speed interval prediction. Shrivastava et al. [41] employed a hybrid framework based on SVM to capture the forecasting uncertainty and results show it can generate high quality prediction intervals. The inherent uncertainty can disturb the decision making and bring risk to wind farm operating, so it is essential to quantify the uncertainty via wind power interval forecasting. Furthermore, point forecasting generates only one forecasting value at each which can simply and directly reflect the future wind power situation [3], while interval forecasting 
produces the upper and lower bounds of the forecasted wind speed at a certain confidence level. To mitigate risks and to improve the management level of the wind power system, point forecasting combining with interval forecasting can make a great contribution.

Considering the superiority of hybrid models, we developed a novel hybrid forecasting system composed of the modules of data preprocessing, forecasting, optimization, and evaluation. Due to inherent volatility and irregularity of the raw wind speed, the data preprocessing module plays a vital part in the system. Furthermore, most previous data preprocessing methods were too primitive to fully mine the characteristics of the wind speed data. Wherefore in this paper, an improved data preprocessing algorithm-Complete Ensemble Empirical Mode Decomposition with Adaptive Noise (CEEMDAN) - is adopted, which can improve the preprocessing effect by adding adaptive white noise among every decomposition. For further improvement, most previous researches employ optimization algorithms to improve the forecasting accuracy. Genetic algorithm (GA) is an effective heuristic algorithm which has shown advantages in wind speed forecasting through the self-improvement mechanism. Wang et al. [27] applied the ensemble empirical mode decomposition (EEMD) and ANN optimized by GA constituting a new hybrid model, which can predict wind speed more accurate than before. However, there is no universal parameter for GA; and it needs to be continually seeking. Furthermore, sometimes a small change of parameters can make a huge difference to the algorithm results and performance. Therefore, GA can be unstable and be susceptible to changes. A. Khosravi [42] compared adaptive neuro-fuzzy inference system (ANFIS) optimized by particle swarm optimization algorithm (PSO) and GA, and the results showed the ANFIS-PSO outperformed the ANFIS-GA, which means the performance of GA is not excellent enough. Additionally, the previous single-objective optimization algorithms, which only focus on improving forecasting accuracy, is not sufficient. So this paper introduces a Multi-Objective Grey Wolf Optimization (MOGWO) algorithm into the optimization module, which focuses on improving the forecasting accuracy as well as enhancing the forecasting stability. Obviously, with the perfection of the model, the model complexity and computing time are increased; therefore, to overcome the aforementioned drawbacks on the basis of guaranteeing the forecasting effect, the forecasting module applies ESN which is simple and fast but of great forecasting capabilities. Finally, the evaluation module evaluates the proposed system based on the experiments of four datasets with different seasons and four datasets with different sites and time intervals. The newly developed hybrid system CEEMDAN-MOGWO-ESN is supposed to balance the superiorities and drawbacks existing in different algorithms and to achieve excellent forecasting results both at wind speed point forecasting and interval forecasting.

The innovation points and contributions of the conducted research are summarized below:

1. Develop a novel hybrid forecasting system for wind speed forecasting. The empirical results indicate the system can integrate advantages of the data preprocessing, optimization, as well as forecasting methods and achieve better forecasting performance in wind speed forecasting.

2. The developed novel forecasting system has strong applicability. This paper employs three experiments to demonstrate that the proposed system can be used for wind speed forecasting with different seasons, different sites, and different time intervals.

3. The proposed hybrid system not only performs well for point forecasting but also implements for interval forecasting. The novel system can quantify the uncertainty as forecasting intervals, and the results indicate the developed system performs well in wind speed interval forecasting.

4. The proposed hybrid system can improve the wind speed forecasting accuracy and stability simultaneously while most previous research can only focus on one side. The experiment results demonstrate that the multi-objective optimization algorithm can not only improve the forecasting accuracy and stability simultaneously but also outperform other optimization algorithms.

5. This paper conducts a comprehensive evaluation of the proposed forecasting system. Ten evaluation metrics, forecasting effectiveness, grey relational analysis, as well as hypothesis testing are combined to demonstrate the advancement of the proposed hybrid forecasting system. 
The structure of the paper is organized as follows: Section 2 presents the relevant theories employed in the proposed model in detail, including the theory of CEEMDAN, MOGWO, and ESN and the evaluation methods. The proposed hybrid system is set forth in Section 3. Section 4 introduces the experiment set and the forecasting results analysis. Several discussions are conducted in Section 5 . Finally, Section 6 concludes the whole paper.

\section{Methodology}

The components of the proposed hybrid system containing the fundamental theories of the decomposition technique CEEMDAN, the forecasting method ESN, the multi-objective optimization algorithm MOGWO, as well as the evaluation methods are presented in this section.

\subsection{Complete Ensemble Empirical Mode Decomposition with Adaptive Noise}

To decrease high computational cost and to eliminate the residual noise of EEMD while alleviating the mode mixing problem of EMD, Torres et al. [43] proposed CEEMDAN by adding the finite time of the adaptive white noise in each decomposition. The distinct advantages of CEEMDAN are as follows: (1) Introduce the noise coefficient vector to control the added noise level for each decomposition; (2) the reconstruction as well as the decomposition is complete and without a noise component; and (3) it is more efficient in the EMD family.

The CEEMDAN computation can be achieved as follows:

Step 1. Adding one white Gaussian noise signal with standard normal distribution, the obtained series can be shown as

$$
s^{i}(n)=s(n)+\varepsilon_{0} v^{i}(n) \quad i=1, \cdots I
$$

where $i$ indicates the trial number, $s(n)$ represents the original series, and $v^{i}(n)$ indicates the added white noise series.

Step 2. Getting the first intrinsic mode function (IMF) by the EMD method:

$$
\operatorname{IMF}_{1}(n)=\left(\sum_{i=1}^{I} I M F_{1}^{i}(n)\right) / I
$$

Step 3. The first residue can be calculated as $(k=1)$ :

$$
r_{1}(n)=s(n)-I M F_{1}(n)
$$

Step 4 . The $k$-th residue can be calculated as

$$
r_{k}(n)=s(n)-I M F_{k}(n) \quad k=2, \cdots K
$$

Step 5. Decompose the residue with added noise to get the $(k+1)$-th IMF:

$$
I M F_{k+1}(n)=\left(\sum_{i=1}^{I} E_{1}\left(r_{k}(n)\right)+\varepsilon_{k} E_{k}\left(v^{i}(n)\right)\right) / I
$$

Step 6. Repeat Steps 4 and 5, until you find out all the IMFs.

In the end, the final residue is

$$
R(n)=s(n)-\sum_{k=1}^{K} I M F_{k}
$$

Thus, the original signal is decomposed into

$$
s(n)=\sum_{k=1}^{K} I M F_{k}+R(n)
$$




\subsection{Echo State Network}

ESN proposed by Jaeger [44] is a new type of recurrent neural network, which is consisted of an input layer, a DR (dynamical reservoir) designed by an interconnected recurrent grid of recurrent neurons, and an output layer. The schematic of ESN is shown in Figure 2D. Different from traditional neural networks, the link weights of the input layer to DR and inside the DR are firstly selected then stay fixed, so merely the DR to output layer link weights need be trained. The ESN output can be expressed as

$$
X(n+1)=F\left(W_{i n} U(n+1)+W X(n)+W_{b a c k} Y(N)\right)
$$

where $U(n+1)$ and $X(n+1)$ represent the input vector and hidden layer state at $(n+1)$; $F$ indicates the hidden layer's activation function; $W_{i n}, W$, and $W_{\text {back }}$ are respectively the input-DR, DR-DR, and output-DR weighting matrix; and the output $Y$ at time $(n+1)$ can be obtained by

$$
Y(n+1)=G\left(W_{\text {out }}\left[U^{T}(n+1), X^{T}(n+1)\right]^{T}\right)
$$

where $W_{\text {out }}$ is the output weight matrix, $W_{\text {out }}=\left(X^{-1} Y\right)^{T}$, and $G$ represents the output layer activation function.

The output weight $W_{\text {out }}$ is the only parameter that needs to be obtained from the training; the $W_{\text {out }}$ can be calculated through the linear regression algorithm:

$$
W_{\text {out }}=\left(X^{-1} Y\right)^{T}
$$

Compared with other methods, ESN shows the following features: (1) The training process of ESN is much faster and simple and (2) ESN shows more advantages in the processing of one-dimension time series.

\subsection{Multi-Objective Grey Wolf Optimization}

The MOGWO proposed by Mirjalili et al. [45] is an advanced optimization algorithm based on the grey wolf optimization (GWO). The GWO simulates the social hunting behavior of grey wolves: the optimal solutions are generated by alpha $(\alpha)$ wolf, the second solutions are generated by beta $(\beta)$ wolf, and the third solutions are generated by delta $(\delta)$ wolf. Furthermore, $\alpha, \beta$, and $\delta$ wolves dominate the exploration for the global optimum, and omega $(\omega)$ wolves, which generate the remainder solutions, just follow them.

The wolves encircle the prey and update its location at the beginning. Equations (11) and (12) simulate the encircling process of the grey wolves [46]:

$$
\begin{gathered}
\vec{G}=\left|\vec{B} \cdot \vec{X}_{p}(t)-\vec{X}(t)\right| \\
\vec{X}(t+1)=\vec{X}_{p}(t)-\vec{K} \cdot \vec{G}
\end{gathered}
$$

where $t$ represents the current iteration, $\vec{X}_{p}$ and $\vec{X}$ denote the location of the prey and grey wolves respectively, and $\vec{B}$ and $\vec{K}$ denote the coefficient vectors and can be calculated as

$$
\begin{gathered}
\vec{K}=2 \vec{k} \cdot \vec{h}_{1}-\vec{k} \\
\vec{B}=2 \cdot \overrightarrow{h_{2}}
\end{gathered}
$$

where $\vec{h}_{1}$ and $\vec{h}_{2}$ are random vectors within $[0,1]$ and $\vec{k}$ decreases from 2 to 0 as the iterations. 
To improve the solution quality, the grey wolves update their positions based on the leading of the first three wolves $(\alpha, \beta$, and $\delta)$. The mechanism of hunting and updating the positions can be simulated as follows:

$$
\begin{aligned}
\vec{G}_{\alpha} & =\left|\overrightarrow{B_{1}} \cdot \overrightarrow{X_{\alpha}}-\vec{X}\right| \\
\vec{G}_{\beta} & =\left|\overrightarrow{B_{2}} \cdot \overrightarrow{X_{\beta}}-\vec{X}\right| \\
\vec{G}_{\delta} & =\left|\overrightarrow{B_{3}} \cdot \vec{X}_{\delta}-\vec{X}\right| \\
\vec{X}_{1} & =\vec{X}_{\alpha}-\overrightarrow{K_{1}} \cdot \vec{G}_{\alpha} \\
\vec{X}_{2} & =\vec{X}_{\beta}-\vec{K}_{2} \cdot \vec{G}_{\beta} \\
\vec{X}_{3} & =\vec{X}_{\delta}-\vec{K}_{3} \cdot \vec{G}_{\delta} \\
\vec{X}(t+1) & =\frac{\vec{X}_{1}+\vec{X}_{2}+\vec{X}_{3}}{3}
\end{aligned}
$$

The MOGWO is developed from the GWO by integrating the two important components: the archive and leader selection strategy [47]. The archive is applied to store the non-dominated solutions generated in each cycle of GWO. The archive management mechanism exploited in MOGWO controls the entering and exiting of the solutions and helps keep the balance of the solutions in the archive. The leader selection strategy plays a vital part in choosing the $\alpha, \beta$, and $\delta$ wolves from the archive by the roulette-wheel method. Additionally, more detailed information about MOGWO can be found in Reference [45].

To improve the forecasting accuracy and to enhance the forecasting stability simultaneously, two objective functions are designed based on the MOGWO. The objective functions are shown in Algorithm 1. For improving the forecasting accuracy, the objective function fitness $s_{1}$ is designed according to the mean absolute error. Meanwhile, to improve the stability, fitness $s_{2}$ is designed via the forecasting error's variance. Because accuracy and stability are both important in wind speed forecasting, the weights of the two objectives are set to be the same.

\subsection{The Forecasting Performance Evaluation}

To evaluate the forecasting performance of the proposed hybrid system in wind speed point forecasting completely, this research designed a complete and scientific evaluation module containing nine appropriate evaluation metrics as well as another two evaluation methods. Moreover, to evaluate the interval forecasting performance of the proposed system, five assessment indices are introduced.

\subsubsection{The Typical Evaluation Metrics}

Various evaluation indicators have been employed to assess the forecasting error by the researchers without a universally accepted standard in comparing different models' forecasting results. Therefore, ten typical evaluation metrics are applied to estimate the forecasting performance, as shown in Table 1. More specifically, the average error of $n$ forecast result (AE) and root mean-square error (RMSE) reflect the forecasting error in a different way. The mean absolute percentage error (MAPE) can reflect the difference between the forecasting and the actual values. The median absolute percentage error (MdAPE) is a measure of statistical dispersion. The fractional bias of $n$ forecast result (FB) shows the number of "under" or "over" forecasting. The index agreement of the forecast result (IA) can compare the performance of different methods since it is dimensionless. The Theil $U$ statistical 1 of forecast result (U1) measures the forecast accuracy, and the Theil $U$ statistical 2 of forecast result (U2) measures the forecast quality. Pearson's correlation coefficient, $r$, is used to measure the correlation relationship between the forecasting and actual values. Except $r$ and $I A$, smaller values of the other error criteria reflect better forecasting performances. 
Table 1. Evaluation Metrics.

\begin{tabular}{|c|c|c|}
\hline Metric & Definition & Equation \\
\hline $\begin{array}{l}M A P E \\
A E\end{array}$ & $\begin{array}{l}\text { Mean absolute percentage error } \\
\text { Average error of } n \text { forecast result }\end{array}$ & $\begin{array}{c}\text { MAPE }=1 / n \times \sum_{i=1}^{n}\left|\left(a_{i}-f_{i}\right) / a_{i}\right| \times 100 \% \\
A E=1 / n \times \sum_{i=1}^{n}\left(a_{i}-f_{i}\right)\end{array}$ \\
\hline RMSE & Root mean-square error & $R M S E=\sqrt{1 / n \times \sum_{i=1}^{n}\left(a_{i}-f_{i}\right)^{2}}$ \\
\hline MdAPE & Median absolute percentage error & $M d A P E=$ median $\left(\left|f_{i}-a_{i} / a_{i}\right| \times 100 \%\right)$ \\
\hline$F B$ & Fractional bias of $n$ forecast result & $F B=2(\bar{a}-\bar{f}) /(\bar{a}+\bar{f})$ \\
\hline$I A$ & Index agreement of the forecast result & $I A=1-\sum_{i=1}^{n}\left(f_{i}-a_{i}\right)^{2} / \sum_{i=1}^{n}\left(\left|f_{i}-\bar{a}\right|+\left|a_{i}+\bar{a}\right|\right)^{2}$ \\
\hline U1 & Theil $U$ statistical 1 of forecast result & $U 1=\sqrt{1 / n \times \sum_{i=1}^{n}\left(a_{i}-f_{i}\right)^{2}} / \sqrt{1 / n \times \sum_{i=1}^{n} f^{2}}+\sqrt{1 / n \times \sum_{i=1}^{n} a_{i}^{2}}$ \\
\hline$U 2$ & Theil $U$ statistical 2 of forecast result & $U 2=\sqrt{1 / n \times \sum_{i=1}^{n}\left(\left(f_{i+1}-a_{i+1}\right) / f_{i}\right)^{2}} / \sqrt{1 / n \times \sum_{i=1}^{n}\left(\left(f_{i+1}-a_{i}\right) / f_{i}\right)^{2}}$ \\
\hline$r$ & Pearson's correlation coefficient & $r=\left(\sum a_{i} f_{i}-\sum a_{i} \sum f_{i} / n\right) / \sqrt{\left(\sum f_{i}{ }^{2}-\left(\sum f_{i}\right)^{2} / n\right)\left(\sum a_{i}{ }^{2}-\left(\sum a_{i}\right)^{2} / n\right)}$ \\
\hline
\end{tabular}


Here, $a_{i}$ and $f_{i}$ respectively signifies the actual value and the forecasting value at time $i, \bar{a}$ and $\bar{f}$ indicate the mean value of the actual value and the forecasting value, respectively, and $n$ represents the size of the sample in this research.

\subsubsection{The Other Two Evaluation Methods}

For further verifying the meliority of the proposed hybrid system in wind speed forecasting, this paper employs the grey relational analysis (GRA) and forecasting effectiveness (FE) [48] to extend the evaluation module. The main thought of GRA is to determine whether the degree of similarity between the forecasting sequence's curve and the original series' curve is high. A higher level of the curve's similarity corresponds to the higher correlation between the corresponding sequences, and vice versa. Wang [49] describes the details of GRA. Moreover, the basic idea of FE is to assess the models' performance by the square sum of the forecasting errors and the mean squared deviation of the forecasting accuracy. A greater FE value indicates the corresponding model performs better and the detailed description is shown by Xiao [50].

\subsubsection{The Assessment Indices for Interval Forecasting}

For short-term wind speed interval forecasting, it is imperative to construct appropriate assessment indices to assess the quality of the prediction intervals. This paper introduces the following five assessment indices based on the literatures. Coverage probability $(\mathrm{CP})$ can indicates the prediction intervals' accuracy, and when CP is confirmed, a smaller average width (AW) corresponds to better performance, accumulated width deviation (AWD) shows the similar effect as AW from another aspect, coverage width criterion (CWC) is used to evaluate the whole quality of the interval forecasting, and score is also an overall evaluation index, where the smaller score indicates better prediction intervals. The detailed expression and calculation are shown in References [51-53].

\section{The Proposed Hybrid Forecasting System}

Hybrid models generally combine two or more algorithms, which is possible to improve the forecasting performance by gathering the excellence of each method [54]. In the proposed forecasting system, it contains the original series' decomposition and recombination, optimization and forecasting, and forecasting result's evaluation. Figure 2 displays the proposed hybrid system's framework, and from Figure 2 the proposed system can be summarized as follows:

Step 1: As shown in Figure 2A, the CEEMDAN decomposes the Raw series into several IMFs, removes the high frequency noise, and recombines the remainders as the Final series.

Step 2: As shown in Figure 2B, the Final series is divided into the training set and testing set, the input-output structure is set as inputting five data and outputting one data.

Step 3: As shown in Figure 2C,D, the MOGWO optimizes the parameters of ESN, employs the training set to train ESN, and updates the parameters of ESN.

Step 4: Employ the testing set to forecast, and the forecasting performance are evaluated by the evaluation metrics shown in Figure 2E.

According to the four steps, the CEEMDAN-MOGWO-ESN hybrid system is constructed; the details are shown in Algorithm 1. Wind speed forecasting can be segmented according to time horizon [55]: short term forecasting is focused on a few seconds to six hours ahead, medium term is from six hours to a day ahead, and long term is exceeding a one day prediction. In this research, wind speed data of $10 \mathrm{~min}$ and $30 \mathrm{~min}$ time intervals are selected to conduct short term forecasting. The forecasting horizon of $10 \mathrm{~min}$ and $30 \mathrm{~min}$ time intervals are $10 \mathrm{~min}$ ahead and $30 \mathrm{~min}$ ahead for one step forecasting. Based on these, this paper conducts short term wind speed forecasting of $10 \mathrm{~min}$ and 30 min time intervals; four experiments using eight datasets are conducted as the illustrative examples to test the performance of the developed system. 


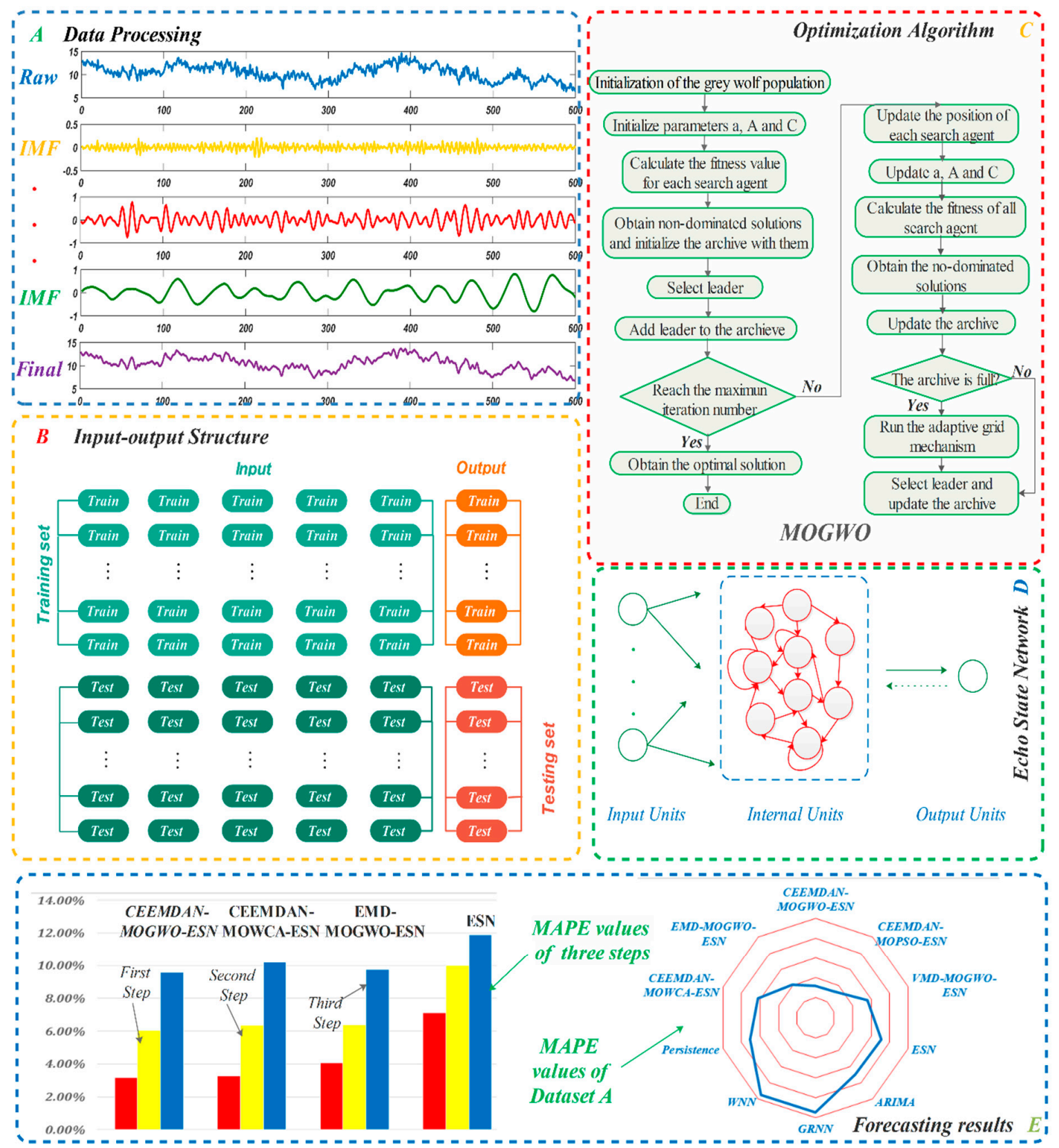

Figure 2. The flowchart of the proposed system: (A) the data preprocessing module; (B) the input-output structure of the developed system; (C) the optimization flowchart of MOGWO; (D) the structure of forecasting method: Echo state network; (E) the forecasting results.

The pseudo-code of the CEEMDAN-MOGWO-ESN algorithm: 


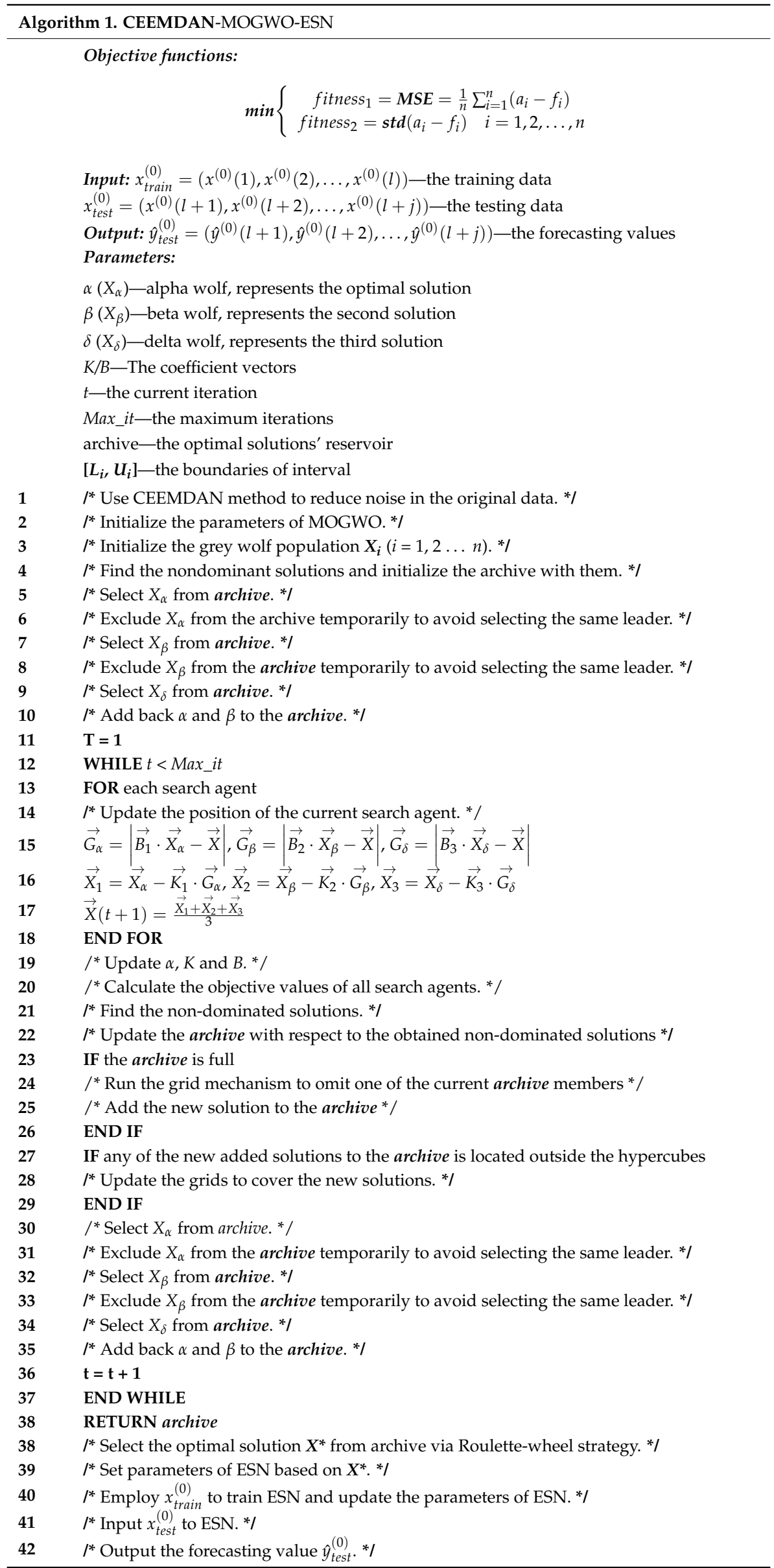




\section{Simulation and Analysis}

For corroborating the proposed hybrid system's superiority of wind speed forecasting, three experiments are conducts as an empirical research employing wind speed data gathered from Penglai wind farms. Specifically, Experiment I applies four datasets in different seasons with 10 min intervals to check the serviceability of the proposed system in different seasons; Experiment II employs four datasets with $10 \mathrm{~min}$ intervals and $30 \mathrm{~min}$ intervals at different sites to confirm the availability of the developed system in the different intervals; and Experiment III adopts GRA and FE to evaluate the hybrid system' further performance. The details of the information are shown as follows.

\subsection{Data Description}

Eight data sets from Penglai in China which has plenty wind energy are considered as cases to inspect the proposed hybrid system's validity in practical applications. Specifically, the datasets A, B, C, and D with 10 min intervals are all gathered at the same data site (Penglai site 1) but from spring, summer, autumn, and winter, respectively. The datasets E with $10 \mathrm{~min}$ intervals as well as datasets $F$ with 30 min intervals are from Penglai site 2, and the datasets $G$ with 10 min intervals and datasets $\mathrm{H}$ with $30 \mathrm{~min}$ intervals are from Penglai site 3 . More specifically, there are altogether 2880 data points in each experiment, in which the training set is composed of the first 2304 observations covered over 16 days and the rest of the 576 data points covered 4 days make up the testing set. For the experiments, Experiment I employs $10 \mathrm{~min}$ wind speed data respectively gathered at Penglai site 1 in spring, summer, autumn and winter, i.e., data sets A, B, C, and D. In Experiment II, $10 \mathrm{~min}$ and $30 \mathrm{~min}$ wind speed data (i.e., datasets $\mathrm{E}$ and datasets $\mathrm{F}$ ) as well as $10 \mathrm{~min}$ and $30 \mathrm{~min}$ data (i.e., data sets $\mathrm{G}$ and datasets $\mathrm{H}$ ) are separately gathered at Penglai site 2 and Penglai site 3. Experiments III and IV are based on Experiment I and Experiment II. The detailed information of the datasets is listed in Table 2.

According to the descriptive statistical characteristic of the experimental data including the average, maximum, median, minimum, and standard deviation, it is clear that the eight wind speed datasets have different features and the experimental data are representative. Indeed, this paper is conducted to test the developed improved forecasting system via numerical simulation and experiments. Thus, this paper employs historical wind speed data to conduct off-line predictions.

\subsection{Experiment I: Comparison of Different Seasons}

In this experiment, the four seasons' wind speed data with $10 \mathrm{~min}$ from Penglai site 1 are employed to verify the developed system's effectiveness in wind speed forecasting. By conducting the experiment with different seasons' data, it can be proved that the seasonal change cannot affect the forecasting ability of the developed system. Moreover, this experiment utilizes four comparative studies to demonstrate the validity of the hybrid system's each component and the progressiveness of the newly proposed hybrid system. Specifically, the first comparison proves the superiority of the employed ESN method in the proposed system by selecting and comparing ESN, GRNN, and WNN; at the second comparison, the advancement of the data preprocessing module is confirmed by comparing the proposed system with EMD-MOGWO-ESN and VMD-MOGWO-ESN; the third comparison is aimed at comparing the proposed system with CEEMDAN-MOPSO-ESN and CEEMDAN-MOWCA-ESN to attest the availability of the optimization method MOGWO; and the last comparison compares the developed hybrid models with the above comparative models as well as Autoregressive Integrated Moving Average (ARIMA) and the persistence model to attest the effectiveness of the developed wind speed forecasting model. The experiment results of the Penglai site 1's wind speed data at four seasons are listed at Table 3 , and the bolded values are the best value of each evaluation metric. The experiment performance can also be more distinctly exhibited by Figure 3; it shows the performance of the proposed forecasting system more intuitively and clearer. Considering Table 3 and Figure 3, the developed CEEMDAN-MOGWO-ESN system gains almost every evaluation metric's best value, which means 
the proposed novel forecasting system outperforms the other benchmark methods in wind speed forecasting. The detailed results and analysis are summarized as follows:

Table 2. The statistic values of all datasets. (Source: Penglai Wind Farm).

\begin{tabular}{|c|c|c|c|c|c|c|}
\hline \multirow{2}{*}{ Dataset } & \multirow{2}{*}{ Season and Interval } & \multicolumn{5}{|c|}{ Statistic Values $(\mathrm{m} / \mathrm{s})$} \\
\hline & & Ave. & Max. & Median & Min. & Std. \\
\hline \multicolumn{7}{|c|}{ Dataset A } \\
\hline All Samples & Spring & 7.6302 & 19.4000 & 7.5000 & 0.8000 & 3.2133 \\
\hline Training Set & & 6.8878 & 14.7000 & 7.0000 & 0.8000 & 2.6857 \\
\hline Testing Set & & 10.5998 & 19.4000 & 10.5000 & 3.2000 & 3.4300 \\
\hline \multicolumn{7}{|c|}{ Dataset B } \\
\hline All Samples & Summer & 4.7082 & 20.5000 & 4.0000 & 0.5000 & 3.0102 \\
\hline Training Set & & 4.4009 & 20.5000 & 3.4000 & 0.5000 & 3.2498 \\
\hline Testing Set & & 5.9375 & 9.7000 & 5.9000 & 3.2000 & 1.0849 \\
\hline \multicolumn{7}{|c|}{ Dataset C } \\
\hline All Samples & Autumn & 5.6823 & 13.2000 & 5.5000 & 0.9000 & 2.2510 \\
\hline Training Set & & 5.7115 & 13.2000 & 5.5000 & 0.9000 & 2.3488 \\
\hline Testing Set & & 5.5653 & 11.7000 & 5.4000 & 2.3000 & 1.8044 \\
\hline \multicolumn{7}{|c|}{ Dataset D } \\
\hline All Samples & Winter & 9.4241 & 18.1000 & 9.2000 & 2.3000 & 2.8516 \\
\hline Training Set & & 9.7592 & 18.1000 & 9.7000 & 2.3000 & 2.9562 \\
\hline Testing Set & & 8.0837 & 12.8000 & 8.1500 & 2.5000 & 1.8609 \\
\hline \multicolumn{7}{|c|}{ Dataset E } \\
\hline All Samples & $10 \mathrm{~min}$ & 9.5891 & 18.7000 & 9.3000 & 2.0000 & 3.2041 \\
\hline Training Set & & 9.9344 & 18.7000 & 9.9000 & 2.0000 & 3.3645 \\
\hline Testing Set & & 8.2078 & 13.3000 & 8.3000 & 2.7000 & 1.9176 \\
\hline \multicolumn{7}{|c|}{ Dataset F } \\
\hline All Samples & $30 \mathrm{~min}$ & 8.2682 & 19.5000 & 8.0000 & 1.3000 & 3.3120 \\
\hline Training Set & & 8.4106 & 18.2000 & 8.1000 & 1.3000 & 3.2873 \\
\hline Testing Set & & 7.6983 & 19.5000 & 7.2000 & 1.5000 & 3.3518 \\
\hline \multicolumn{7}{|c|}{ Dataset G } \\
\hline All Samples & $10 \mathrm{~min}$ & 7.3951 & 18.2000 & 6.6000 & 1.0000 & 3.2506 \\
\hline Training Set & & 7.7470 & 18.2000 & 7.1000 & 1.0000 & 3.3104 \\
\hline Testing Set & & 5.9877 & 12.4000 & 5.3000 & 1.2000 & 2.5563 \\
\hline \multicolumn{7}{|c|}{ Dataset $\mathrm{H}$} \\
\hline All Samples & $30 \mathrm{~min}$ & 7.5068 & 18.3000 & 7.2000 & 0.9000 & 3.1448 \\
\hline Training Set & & 7.7140 & 18.3000 & 7.5000 & 0.9000 & 3.2272 \\
\hline Testing Set & & 6.6783 & 14.7000 & 6.5000 & 0.9000 & 2.6350 \\
\hline
\end{tabular}


Table 3. Results of Experiment I.

\begin{tabular}{|c|c|c|c|c|c|c|c|c|c|c|c|}
\hline Dataset & Model & $A E$ & MAPE & RMSE & $M d A P E$ & $F B$ & $r$ & $I A$ & U1 & $U 2$ & TIME (s) \\
\hline \multicolumn{12}{|c|}{ Spring } \\
\hline \multirow{10}{*}{ Dataset A } & Persistence Model & -0.0069 & 7.0651 & 0.9094 & 0.0531 & 0.0007 & 0.9650 & 0.9822 & 0.0410 & 0.7096 & 0.0105 \\
\hline & ARIMA & -0.0295 & 6.9594 & 0.8940 & 0.0547 & 0.0028 & 0.9657 & 0.9824 & 0.0403 & 0.7072 & 211.2213 \\
\hline & GRNN & -0.5289 & 9.4339 & 1.4625 & 0.0749 & 0.0514 & 0.9287 & 0.9420 & 0.0680 & 0.8123 & 1.2176 \\
\hline & WNN & -0.6424 & 9.5246 & 1.7733 & 0.0735 & 0.0627 & 0.8887 & 0.9099 & 0.0831 & 0.8211 & 2.7442 \\
\hline & ESN & -0.1029 & 7.1156 & 0.9258 & 0.0548 & 0.0098 & 0.9636 & 0.9810 & 0.0419 & 0.7158 & 0.9458 \\
\hline & EMD-MOGWO-ESN & -0.0193 & 4.0520 & 0.5122 & 0.0331 & 0.0018 & 0.9889 & 0.9944 & 0.0231 & 0.5817 & 189.2712 \\
\hline & VMD-MOGWO-ESN & -0.0108 & 5.5978 & 0.6921 & 0.0413 & 0.0010 & 0.9801 & 0.9892 & 0.0312 & 0.8985 & 238.5490 \\
\hline & CEEMDAN-MOGWO-ESN & -0.0294 & 3.1490 & 0.4182 & 0.0242 & 0.0028 & 0.9927 & 0.9962 & 0.0189 & 0.4680 & 449.3265 \\
\hline & CEEMDAN-MOPSO-ESN & -0.1164 & 6.2568 & 0.8000 & 0.0467 & 0.0111 & 0.9733 & 0.9857 & 0.0363 & 0.6756 & 480.3966 \\
\hline & CEEMDAN-MOWCA-ESN & 0.0049 & 3.2530 & 0.4356 & 0.0247 & -0.0005 & 0.9920 & 0.9960 & 0.0196 & 0.4799 & 308.7396 \\
\hline \multicolumn{12}{|c|}{ Summer } \\
\hline \multirow{10}{*}{ Dataset B } & Persistence Model & -0.0004 & 6.3072 & 0.4874 & 0.0497 & 0.0001 & 0.8987 & 0.9473 & 0.0403 & 0.7153 & 0.0130 \\
\hline & ARIMA & -0.0011 & 7.6967 & 0.5939 & 0.0605 & 0.0002 & 0.8504 & 0.9209 & 0.0491 & 0.7759 & 253.2662 \\
\hline & GRNN & -0.1677 & 7.4153 & 0.5648 & 0.0626 & 0.0286 & 0.8686 & 0.9227 & 0.0474 & 0.7941 & 1.2075 \\
\hline & WNN & -0.1202 & 6.5569 & 0.5125 & 0.0512 & 0.0204 & 0.8884 & 0.9366 & 0.0428 & 0.7500 & 2.7869 \\
\hline & ESN & -0.0979 & 6.4067 & 0.4953 & 0.0523 & 0.0166 & 0.8957 & 0.9430 & 0.0413 & 0.7358 & 0.9696 \\
\hline & EMD-MOGWO-ESN & -0.0267 & 3.8015 & 0.2827 & 0.0330 & 0.0045 & 0.9658 & 0.9824 & 0.0234 & 0.6034 & 193.5132 \\
\hline & VMD-MOGWO-ESN & -0.0169 & 4.8781 & 0.3576 & 0.0395 & 0.0028 & 0.9471 & 0.9682 & 0.0296 & 0.8462 & 196.6962 \\
\hline & CEEMDAN-MOGWO-ESN & -0.0185 & 3.0051 & 0.2321 & 0.0238 & 0.0031 & 0.9770 & 0.9879 & 0.0192 & 0.4789 & 312.1663 \\
\hline & CEEMDAN-MOPSO-ESN & -0.0332 & 6.0292 & 0.4465 & 0.0511 & 0.0056 & 0.9144 & 0.9554 & 0.0370 & 0.6961 & 456.7801 \\
\hline & CEEMDAN-MOWCA-ESN & -0.0287 & 3.1738 & 0.2425 & 0.0262 & 0.0048 & 0.9750 & 0.9870 & 0.0201 & 0.5069 & 337.8480 \\
\hline
\end{tabular}


Table 3. Cont.

\begin{tabular}{|c|c|c|c|c|c|c|c|c|c|c|c|}
\hline Dataset & Model & $A E$ & MAPE & RMSE & MdAPE & $F B$ & $r$ & $I A$ & U1 & $U 2$ & TIME (s) \\
\hline \multicolumn{12}{|c|}{ Autumn } \\
\hline \multirow{10}{*}{ Dataset C } & Persistence Model & 0.0021 & 6.5623 & 0.4371 & 0.0515 & -0.0004 & 0.9707 & 0.9851 & 0.0372 & 0.7878 & 0.0168 \\
\hline & ARIMA & 0.0293 & 7.5086 & 0.4937 & 0.0592 & -0.0052 & 0.9622 & 0.9806 & 0.0420 & 0.8218 & 217.7421 \\
\hline & GRNN & -0.0163 & 6.8788 & 0.4602 & 0.0528 & 0.0029 & 0.9670 & 0.9829 & 0.0393 & 0.8027 & 1.2009 \\
\hline & WNN & 0.1651 & 7.9610 & 0.4840 & 0.0556 & -0.0291 & 0.9700 & 0.9800 & 0.0408 & 0.8017 & 2.8116 \\
\hline & ESN & 0.0004 & 6.5827 & 0.4344 & 0.0509 & -0.0001 & 0.9707 & 0.9850 & 0.0370 & 0.7826 & 1.0093 \\
\hline & EMD-MOGWO-ESN & 0.0058 & 3.8554 & 0.2464 & 0.0303 & -0.0010 & 0.9907 & 0.9953 & 0.0210 & 0.6131 & 180.8671 \\
\hline & VMD-MOGWO-ESN & -0.0033 & 5.5412 & 0.3605 & 0.0406 & 0.0006 & 0.9809 & 0.9893 & 0.0308 & 0.8689 & 188.5874 \\
\hline & CEEMDAN-MOGWO-ESN & 0.0031 & 3.0618 & 0.2026 & 0.0230 & -0.0005 & 0.9937 & 0.9968 & 0.0172 & 0.4978 & 435.9351 \\
\hline & CEEMDAN-MOPSO-ESN & 0.0055 & 5.8461 & 0.3848 & 0.0451 & -0.0010 & 0.9771 & 0.9882 & 0.0328 & 0.6989 & 542.4545 \\
\hline & CEEMDAN-MOWCA-ESN & 0.0072 & 4.3007 & 0.2855 & 0.0305 & -0.0013 & 0.9874 & 0.9936 & 0.0243 & 0.6066 & 305.6523 \\
\hline \multicolumn{12}{|c|}{ Winter } \\
\hline \multirow{10}{*}{ Dataset D } & Persistence Model & 0.0129 & 5.3605 & 0.6108 & 0.0419 & -0.0016 & 0.9437 & 0.9711 & 0.0368 & 0.7583 & 0.0113 \\
\hline & ARIMA & 0.0238 & 7.2111 & 0.8077 & 0.0559 & -0.0029 & 0.9025 & 0.9492 & 0.0486 & 0.8668 & 219.5740 \\
\hline & GRNN & 0.0391 & 5.8629 & 0.6283 & 0.0438 & -0.0048 & 0.9387 & 0.9673 & 0.0379 & 0.8194 & 1.8741 \\
\hline & WNN & 0.1930 & 6.2114 & 0.6311 & 0.0460 & -0.0236 & 0.9439 & 0.9677 & 0.0377 & 0.8097 & 3.0436 \\
\hline & ESN & 0.0403 & 5.3931 & 0.5937 & 0.0396 & -0.0050 & 0.9456 & 0.9714 & 0.0358 & 0.7768 & 0.9342 \\
\hline & EMD-MOGWO-ESN & 0.0199 & 3.4907 & 0.3715 & 0.0288 & -0.0025 & 0.9790 & 0.9893 & 0.0224 & 0.6482 & 192.9672 \\
\hline & VMD-MOGWO-ESN & 0.0124 & 4.3556 & 0.4658 & 0.0327 & -0.0015 & 0.9678 & 0.9820 & 0.0281 & 0.8091 & 199.8609 \\
\hline & CEEMDAN-MOGWO-ESN & 0.0098 & 2.6180 & 0.3053 & 0.0206 & -0.0012 & 0.9858 & 0.9928 & 0.0184 & 0.5426 & 434.4075 \\
\hline & CEEMDAN-MOPSO-ESN & 0.0374 & 4.7831 & 0.5222 & 0.0363 & -0.0046 & 0.9584 & 0.9786 & 0.0314 & 0.7165 & 558.1496 \\
\hline & CEEMDAN-MOWCA-ESN & 0.0034 & 2.7343 & 0.3170 & 0.0206 & -0.0004 & 0.9847 & 0.9922 & 0.0191 & 0.5528 & 318.4237 \\
\hline
\end{tabular}




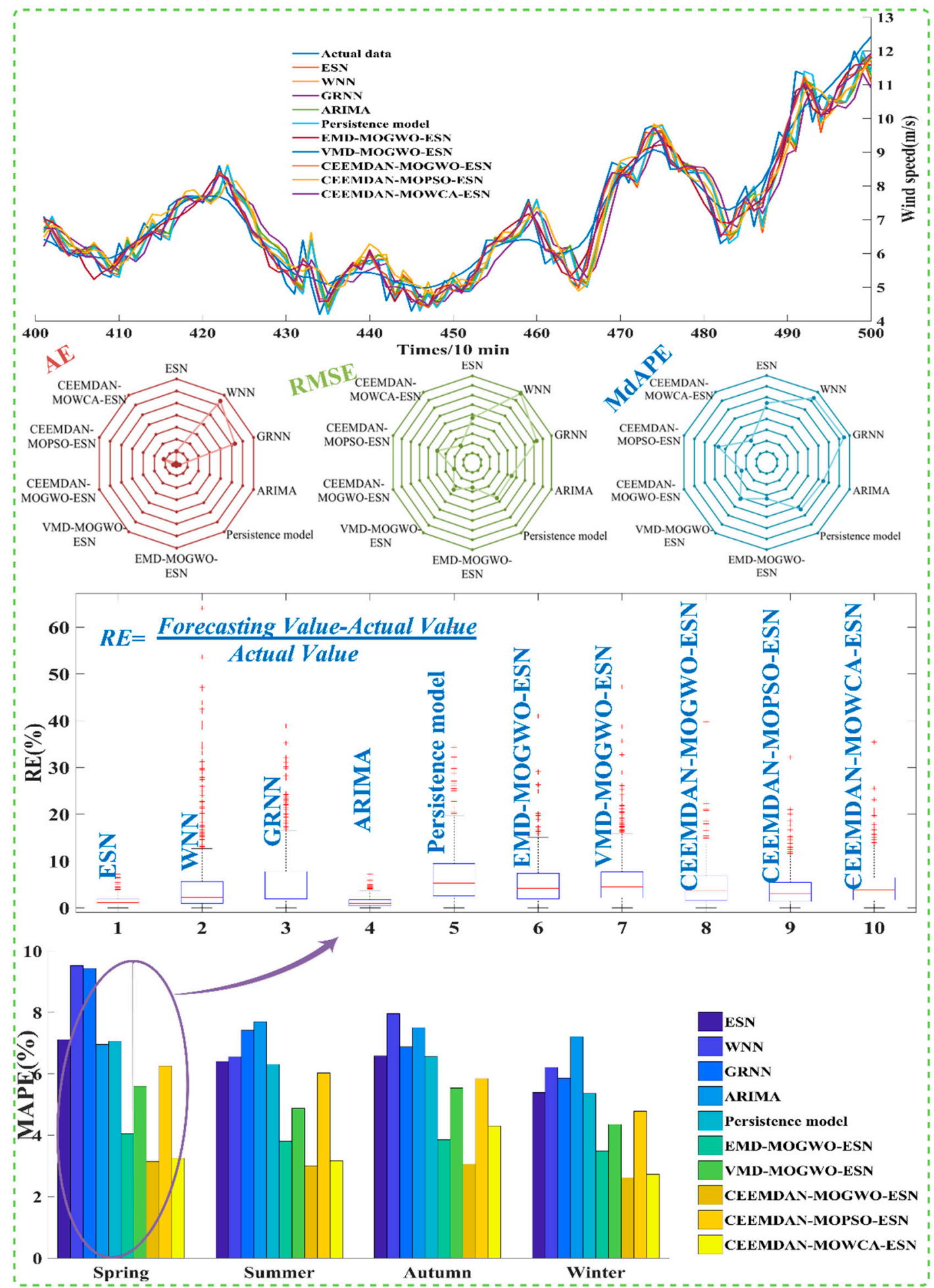

Figure 3. Forecasting results of the proposed system in four seasons.

(1) Comparing ESN with other artificial intelligence methods (GRNN and WNN), it can be found that ESN has a better forecasting ability than GRNN and WNN. Moreover, the runtime of ESN reduces significantly compared with GRNN and WNN. Therefore, the used ESN can not only improve the forecasting accuracy but also reduce the developed system's runtime, which means the forecast method used in the developed forecasting system is preeminent.

(2) From comparing the developed forecasting system with other hybrid methods (EMD-MOGWOESN and VMD-MOGWO-ESN), it is obvious that the proposed CEEMDAN-MOGWO-ESN can improve 
forecasting accuracy significantly. In this comparison, only the data preprocessing methods are different, so the effort of the developed system's data preprocessing method is proved. It means that the data preprocessing method CEEMDAN used in the proposed system can conduct effective data preprocessing and contribute to improving the forecasting accuracy.

(3) By comparing the developed system with contract models (CEEMDAN-MOPSO-ESN and CEEMDAN-MOWCA-ESN), the advancement of the multi-object optimization algorithm used in the proposed system is confirmed. Only the optimization algorithm is different in the contract models; therefore the discrepancy in the forecasting ability of these models results from the optimization algorithm changes. The developed system outperforms the contract models in forecasting, so the MOGWO outperforms the contract optimization algorithm (MOPSO and MOWCA). It reveals that the multi-object optimization algorithm MOGWO can enhance the forecasting effectiveness.

(4) The comparisons between the developed system and other comparative methods have clearly confirmed the advancement of the proposed hybrid system in wind speed forecasting. Each component has a certain enhancement for the forecasting ability respectively and can be added up to promote the forecasting performance as a hybrid system. Furthermore, by comparing with ARIMA, the persistence model, and other models, the developed forecasting system's superiority is revealed absolutely.

(5) When comparing the developed system with other methods, the developed system prevails over the comparative methods in each case, which means that the proposed hybrid system performs better in wind speed forecasting no matter which season. For the developed system, its MAPE values are $3.1490 \%, 3.0051 \%, 3.0618 \%$, and $2.6180 \%$ at spring, summer, autumn, and winter, respectively. Therefore, the developed hybrid system is a reliable forecasting method, and its forecasting ability is not affected by seasonal factors.

Remark: For all seasons' datasets in Penglai site 1, the developed hybrid system obtains nearly all the best values of all the evaluation indexes comparing with the other comparative models. Therefore, the developed system has a reliable forecasting ability owing to the excellent hybrid strategy; moreover, it can forecast wind speed effectively and be not affected by seasonal factors.

\subsection{Experiment II: Comparison of a Different Time Interval}

For further validating the outstanding forecasting ability of the proposed system in this paper and demonstrating the validity of the proposed forecasting system when forecasting wind speeds of different time intervals or diverse data features, it employs $10 \mathrm{~min}$ and $30 \mathrm{~min}$ data form Penglai site 2 and Penglai site 3 for the experiment. In Experiment II, there are two comparative studies organized. The first comparative study employs two sets of $10 \mathrm{~min}$ and $30 \mathrm{~min}$ wind speed series' comparisons to affirm the developed system's validity in forecasting the wind speed of different time intervals. While the other comparative study conducts two sets of comparison between the wind speed data with the same intervals, it collected from different sites to prove the developed system can perform well no matter which site the data is gathered from. Each comparison is expressed by testing the developed system and benchmark models such as ARIMA, GRNN, EMD-MOGWO-ESN, CEEMDAN-MOPSO-ESN, and others. The performance of this experiment is distinctly shown in Figure 4, and the results of Experiment II are presented in Tables 4 and 5. According to Tables 4 and 5, and Figure 4, the excellent forecasting performance of the developed system can be demonstrated. The detailed results and analysis are summarized as follows:

(1) In the first comparative study, comparing the wind speed forecasting performance of the proposed system with other comparative methods at $10 \mathrm{~min}$ and $30 \mathrm{~min}$ reveals the developed system's wonderful forecasting performance in different time interval. Such as, the $10 \mathrm{~min}$ data's MAPE of the proposed system at Penglai site 3 is $2.6276 \%$, the ESN is $5.1874 \%$, the GRNN is $6.6787 \%$, the ARIMA is $5.8801 \%$, the VMD-MOGWO-ESN is $4.1352 \%$ and the CEEMDAN-GWO-ESN is $2.6891 \%$ which performances the best in the comparative models. The comparative study's consequences indicate that the proposed system can forecast well at different time intervals. 
(2) The second comparative study conducted with wind speed of the same time interval but different characteristics reveals the developed system's excellent forecasting performance. For a $10 \mathrm{~min}$ wind speed forecasting, the developed system performs the best at dataset $\mathrm{E}$ and dataset $\mathrm{G}$ and the other compared models also obtain good forecasting results. But for a wind speed with a $30 \mathrm{~min}$ interval, the excellent forecasting ability of the developed system has emerged. The MAPE of the developed forecasting system is $4.9663 \%$ and $5.9178 \%$ at dataset $\mathrm{F}$ and dataset $\mathrm{H}$, the developed system not only gains the best MAPE value but also achieves the least discrepancy of the forecasting performances. As to the compared models, for example, the MAPE values of EMD-MOGWO-ESN, CEEMDAN-MOWCA-ESN, GRNN, and ARIMA are $6.1778 \%, 5.8910 \%, 11.8085 \%$, and $12.4851 \%$ and are $8.2231 \%, 6.2213 \%, 16.8312 \%$, and $14.837 \%$ at dataset $\mathrm{F}$ and dataset $\mathrm{H}$, respectively.

(3) When comparing the forecasting performance of the proposed system and other methods at different sites and time intervals, it can be found that the developed system can forecast well beyond the limitations of time and space. For example, the developed system's MAPE values are $2.6227 \%$ of dataset $E, 4.9663 \%$ of dataset $F, 2.6276 \%$ of dataset $G$, and $5.9178 \%$ of dataset $H$, and the hybrid strategy increases the forecasting accuracy by $2.7875 \%, 5.7176 \%, 2.5596 \%$, and $7.8702 \%$, respectively. This indicates that the developed hybrid system can improve wind speed forecasting effects effectively and obtain high forecasting ability.

Remark: The comparison results of the cases from different time intervals and different wind sites certify the contribution of the proposed hybrid strategy and advancement of the proposed forecasting system. Furthermore, based on the various evaluation metrics, it is proved that the developed system can perform well without the influence of the time intervals and sites. 


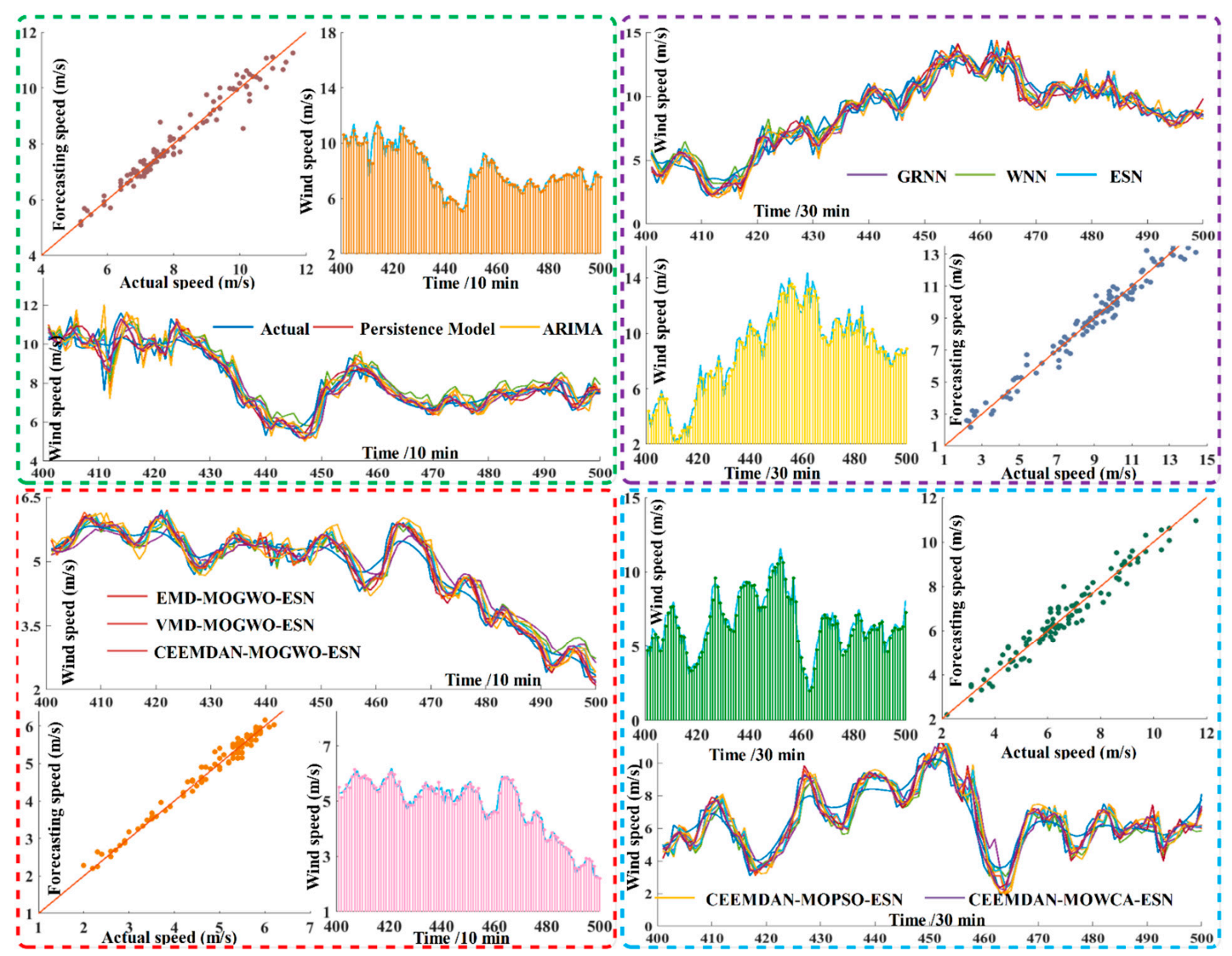

Figure 4. Forecasting performance of the proposed system at different time intervals. 
Table 4. Forecasting performance at Penglai site 2.

\begin{tabular}{|c|c|c|c|c|c|c|c|c|c|c|c|}
\hline Dataset & Model & $A E$ & MAPE & RMSE & $M d A P E$ & $F B$ & $r$ & $I A$ & U1 & $U 2$ & TIME (s) \\
\hline \multicolumn{12}{|c|}{10 min } \\
\hline \multirow{10}{*}{ Dataset E } & Persistence Model & 0.0125 & 5.3793 & 0.6434 & 0.0427 & -0.0015 & 0.9411 & 0.9697 & 0.0382 & 0.7518 & 0.0096 \\
\hline & ARIMA & 0.0375 & 7.2781 & 0.8497 & 0.0540 & -0.0046 & 0.8979 & 0.9465 & 0.0503 & 0.8580 & 224.4170 \\
\hline & GRNN & 0.0360 & 5.9703 & 0.6648 & 0.0439 & -0.0044 & 0.9352 & 0.9654 & 0.0394 & 0.8101 & 1.1879 \\
\hline & WNN & 0.3095 & 7.3064 & 0.7158 & 0.0541 & -0.0370 & 0.9397 & 0.9589 & 0.0418 & 0.8368 & 2.8472 \\
\hline & ESN & 0.0289 & 5.4102 & 0.6242 & 0.0405 & -0.0035 & 0.9432 & 0.9704 & 0.0370 & 0.7665 & 0.8773 \\
\hline & EMD-MOGWO-ESN & 0.0170 & 3.3775 & 0.3937 & 0.0270 & -0.0021 & 0.9777 & 0.9886 & 0.0234 & 0.6803 & 175.0164 \\
\hline & VMD-MOGWO-ESN & 0.0058 & 3.8882 & 0.4579 & 0.0274 & -0.0007 & 0.9703 & 0.9839 & 0.0272 & 0.8890 & 180.0621 \\
\hline & CEEMDAN-MOGWO-ESN & 0.0035 & 2.6227 & 0.3113 & 0.0190 & -0.0004 & 0.9861 & 0.9929 & 0.0185 & 0.5264 & 433.6128 \\
\hline & CEEMDAN-MOPSO-ESN & 0.0512 & 4.8447 & 0.5500 & 0.0360 & -0.0062 & 0.9565 & 0.9775 & 0.0326 & 0.7108 & 446.8051 \\
\hline & CEEMDAN-MOWCA-ESN & 0.0094 & 2.8024 & 0.3273 & 0.0216 & -0.0011 & 0.9847 & 0.9922 & 0.0194 & 0.5518 & 505.6086 \\
\hline \multicolumn{12}{|c|}{$30 \mathrm{~min}$} \\
\hline \multirow{10}{*}{ Dataset F } & Persistence Model & 0.0004 & 10.3314 & 0.9544 & 0.0736 & 0.0000 & 0.9599 & 0.9796 & 0.0569 & 0.7678 & 0.0216 \\
\hline & ARIMA & -0.0166 & 12.4851 & 1.1319 & 0.0892 & 0.0022 & 0.9427 & 0.9705 & 0.0677 & 0.8307 & 217.0166 \\
\hline & GRNN & 0.0163 & 11.8085 & 1.0037 & 0.0834 & -0.0021 & 0.9551 & 0.9756 & 0.0601 & 0.7950 & 1.9596 \\
\hline & WNN & 0.2123 & 12.9329 & 1.0253 & 0.0846 & -0.0273 & 0.9584 & 0.9733 & 0.0610 & 0.8060 & 2.8900 \\
\hline & ESN & 0.0389 & 10.6839 & 0.9373 & 0.0728 & -0.0051 & 0.9608 & 0.9792 & 0.0560 & 0.7506 & 0.8695 \\
\hline & EMD-MOGWO-ESN & -0.0093 & 6.1778 & 0.5711 & 0.0478 & 0.0012 & 0.9856 & 0.9926 & 0.0341 & 0.6243 & 173.2602 \\
\hline & VMD-MOGWO-ESN & 0.0022 & 9.6131 & 0.7905 & 0.0621 & -0.0003 & 0.9730 & 0.9851 & 0.0474 & 0.8869 & 175.1273 \\
\hline & CEEMDAN-MOGWO-ESN & 0.0121 & 4.9663 & 0.4550 & 0.0347 & -0.0016 & 0.9910 & 0.9953 & 0.0272 & 0.5196 & 403.4519 \\
\hline & CEEMDAN-MOPSO-ESN & 0.0396 & 9.0774 & 0.7930 & 0.0638 & -0.0051 & 0.9721 & 0.9854 & 0.0473 & 0.6756 & 569.2328 \\
\hline & CEEMDAN-MOWCA-ESN & 0.0187 & 5.8910 & 0.5293 & 0.0427 & -0.0024 & 0.9877 & 0.9936 & 0.0316 & 0.5498 & 572.0835 \\
\hline
\end{tabular}


Table 5. Forecasting performance at Penglai site 3.

\begin{tabular}{|c|c|c|c|c|c|c|c|c|c|c|c|}
\hline Dataset & Model & $A E$ & MAPE & RMSE & $M d A P E$ & $F B$ & $r$ & $I A$ & U1 & $U 2$ & TIME (s) \\
\hline \multicolumn{12}{|c|}{$10 \mathrm{~min}$} \\
\hline \multirow{10}{*}{ Dataset $G$} & Persistence Model & 0.0019 & 4.9369 & 0.4111 & 0.0417 & -0.0003 & 0.9868 & 0.9933 & 0.0313 & 0.6985 & 0.0129 \\
\hline & ARIMA & 0.0113 & 5.8801 & 0.4790 & 0.0470 & -0.0019 & 0.9819 & 0.9908 & 0.0365 & 0.7496 & 224.4170 \\
\hline & GRNN & 0.0150 & 6.6787 & 0.4973 & 0.0521 & -0.0025 & 0.9807 & 0.9898 & 0.0380 & 0.7847 & 1.1958 \\
\hline & WNN & -0.0369 & 6.0838 & 0.4501 & 0.0418 & 0.0061 & 0.9854 & 0.9915 & 0.0346 & 0.8083 & 2.7610 \\
\hline & ESN & 0.0250 & 5.1874 & 0.4140 & 0.0414 & -0.0041 & 0.9866 & 0.9931 & 0.0315 & 0.7079 & 0.9250 \\
\hline & EMD-MOGWO-ESN & 0.0076 & 2.9281 & 0.2378 & 0.0245 & -0.0013 & 0.9956 & 0.9978 & 0.0181 & 0.5539 & 184.8949 \\
\hline & VMD-MOGWO-ESN & -0.0019 & 4.1352 & 0.3215 & 0.0341 & 0.0003 & 0.9920 & 0.9958 & 0.0245 & 0.8627 & 203.6287 \\
\hline & CEEMDAN-MOGWO-ESN & 0.0067 & 2.6276 & 0.2001 & 0.0203 & -0.0011 & 0.9969 & 0.9984 & 0.0153 & 0.5064 & 437.2306 \\
\hline & CEEMDAN-MOPSO-ESN & 0.0084 & 5.0573 & 0.3870 & 0.0396 & -0.0014 & 0.9882 & 0.9940 & 0.0295 & 0.6870 & 695.8269 \\
\hline & CEEMDAN-MOWCA-ESN & 0.0115 & 3.6828 & 0.2898 & 0.0296 & -0.0019 & 0.9934 & 0.9967 & 0.0221 & 0.5832 & 482.3285 \\
\hline \multicolumn{12}{|c|}{$30 \mathrm{~min}$} \\
\hline \multirow{10}{*}{ Dataset $H$} & Persistence Model & -0.0053 & 12.9621 & 1.0334 & 0.0940 & 0.0008 & 0.9239 & 0.9608 & 0.0719 & 0.7000 & 0.0125 \\
\hline & ARIMA & 0.0250 & 14.8370 & 1.1431 & 0.1005 & -0.0037 & 0.9048 & 0.9505 & 0.0796 & 0.7215 & 217.0166 \\
\hline & GRNN & 0.0466 & 16.8312 & 1.1171 & 0.1067 & -0.0069 & 0.9068 & 0.9495 & 0.0780 & 0.8412 & 2.1636 \\
\hline & WNN & -0.0775 & 17.8125 & 1.1096 & 0.1063 & 0.0117 & 0.9084 & 0.9502 & 0.0781 & 0.9446 & 2.8652 \\
\hline & ESN & 0.0368 & 13.7880 & 1.0290 & 0.0937 & -0.0055 & 0.9219 & 0.9590 & 0.0717 & 0.6993 & 1.1308 \\
\hline & EMD-MOGWO-ESN & 0.0268 & 8.2231 & 0.6164 & 0.0645 & -0.0040 & 0.9727 & 0.9861 & 0.0429 & 0.5817 & 174.0264 \\
\hline & VMD-MOGWO-ESN & -0.0037 & 10.7047 & 0.7456 & 0.0749 & 0.0005 & 0.9614 & 0.9777 & 0.0522 & 0.8418 & 170.0751 \\
\hline & CEEMDAN-MOGWO-ESN & 0.0089 & 5.9178 & 0.4612 & 0.0457 & -0.0013 & 0.9847 & 0.9922 & 0.0321 & 0.4449 & 451.9599 \\
\hline & CEEMDAN-MOPSO-ESN & 0.0938 & 11.9054 & 0.8586 & 0.0837 & -0.0139 & 0.9469 & 0.9724 & 0.0595 & 0.6612 & 512.7695 \\
\hline & CEEMDAN-MOWCA-ESN & 0.0151 & 6.2213 & 0.4812 & 0.0462 & -0.0023 & 0.9834 & 0.9915 & 0.0335 & 0.4770 & 480.7268 \\
\hline
\end{tabular}




\subsection{Experiment III: Further Evaluating the Proposed System}

Though the evaluation metrics listed in Table 1 has been applied to attest the developed system's forecasting performance, the evaluation still needs to be further conducted. Thus, FE and GRA are added to demonstrate the super-excellence of the developed hybrid system in wind speed forecasting to a greater degree. FE indicates the forecasting accuracy while GRA represents the forecasting performance by reflecting the correlation between the forecasting values and the actual values. Here all datasets are applied to realize the FE and GRA test, further verifying the results of Experiment I and Experiment II. The experiment results are listed at Table 6, where the bigger value of the result indicates the better forecasting performance and the best results appear in bold. From Table 6, both FE values and GRA values of the proposed system are the greatest among all the models in the experiment, which means the developed forecasting system prevails over the comparative methods in all cases.

Remark: The FE and GRA further validate that the proposed system is a reliable and valid tool to forecast wind speed compared with other methods. Moreover, the forecasting accuracy and the relevance between forecasting values and actual values of the developed system are also demonstrated. In summary, the developed system can perform well in wind speed forecasting.

\subsection{Experiment IV: Wind Speed Interval Forecasting}

In this experiment, we use dataset $\mathrm{D}$ and $\mathrm{E}$ as examples to verify the interval forecasting performance of the developed system. To confirm the distribution of the construct prediction intervals, this paper employs four methods fitting the error distribution. As the results shown in Table 7 and Figure 5a, the Logistic distribution is the best fitted one and the Gaussian distribution is close to the Logistic distribution. Thus, we construct the prediction intervals via the Logistic distribution and evaluate the intervals by the assessment indices such as CP, AW, AWD, CWC, and score at 95\%, $90 \%, 85 \%, 80 \%$, and $70 \%$ confidence levels, respectively. On the account of the similar fitting results between the Logistic distribution and Gaussian distribution, we contract the two distributions at the same situation. The results are shown in Table 8 and Figure 5b; from the results, we can find that the developed system performs better in wind speed interval forecasting when fitting the distribution by Logistic rather than the Gaussian distribution. Figure 5 intuitively shows that the developed system possesses an excellent wind speed interval forecasting ability. 
Table 6. Forecasting results evaluated by the forecasting effectiveness (FE) and grey relational degree (GRD).

\begin{tabular}{|c|c|c|c|c|c|c|c|c|c|c|c|c|}
\hline \multirow[t]{2}{*}{ Models } & \multicolumn{3}{|c|}{ Dataset A } & \multicolumn{3}{|c|}{ Dataset B } & \multicolumn{3}{|c|}{ Dataset C } & \multicolumn{3}{|c|}{ Dataset D } \\
\hline & FE-1 & FE-2 & GRD & FE-1 & FE-2 & GRD & FE-1 & FE-2 & GRD & FE-1 & FE-2 & GRD \\
\hline Persistence Model & 0.9295 & 0.8691 & 0.8871 & 0.9368 & 0.8859 & 0.7751 & 0.9345 & 0.8774 & 0.7919 & 0.9465 & 0.9021 & 0.8653 \\
\hline ARIMA & 0.9306 & 0.8702 & 0.8875 & 0.9227 & 0.8602 & 0.7456 & 0.9251 & 0.8616 & 0.7689 & 0.9280 & 0.8688 & 0.8336 \\
\hline GRNN & 0.9057 & 0.8304 & 0.8495 & 0.9258 & 0.8721 & 0.7470 & 0.9314 & 0.8712 & 0.7823 & 0.9414 & 0.8910 & 0.8589 \\
\hline WNN & 0.9049 & 0.8230 & 0.8404 & 0.9258 & 0.8721 & 0.7470 & 0.9202 & 0.8478 & 0.7856 & 0.9378 & 0.8830 & 0.8644 \\
\hline ESN & 0.9295 & 0.8688 & 0.8857 & 0.9360 & 0.8871 & 0.7712 & 0.9345 & 0.8781 & 0.7897 & 0.9457 & 0.8991 & 0.8670 \\
\hline EMD-MOGWO-ESN & 0.9596 & 0.9274 & 0.9290 & 0.9620 & 0.9349 & 0.8413 & 0.9614 & 0.9288 & 0.8579 & 0.9651 & 0.9395 & 0.9034 \\
\hline VMD-MOGWO-ESN & 0.9442 & 0.8904 & 0.9101 & 0.9514 & 0.9153 & 0.8155 & 0.9447 & 0.8943 & 0.8203 & 0.9565 & 0.9172 & 0.8882 \\
\hline CEEMDAN-MOGWO-ESN & 0.9686 & 0.9409 & 0.9434 & 0.9699 & 0.9451 & 0.8697 & 0.9694 & 0.9423 & 0.8838 & 0.9738 & 0.9508 & 0.9252 \\
\hline CEEMDAN-MOPSO-ESN & 0.9376 & 0.8835 & 0.8997 & 0.9397 & 0.8947 & 0.7780 & 0.9416 & 0.8890 & 0.8056 & 0.9522 & 0.9148 & 0.8771 \\
\hline \multirow[t]{3}{*}{ CEEMDAN-MOWCA-ESN } & 0.9676 & 0.9385 & 0.9419 & 0.9682 & 0.9435 & 0.8639 & 0.9570 & 0.9188 & 0.8468 & 0.9727 & 0.9484 & 0.9230 \\
\hline & \multicolumn{3}{|c|}{ Dataset E } & \multicolumn{3}{|c|}{ Dataset F } & \multicolumn{3}{|c|}{ Dataset G } & \multicolumn{3}{|c|}{ Dataset $\mathbf{H}$} \\
\hline & FE-1 & FE-2 & GRD & FE-1 & FE-2 & GRD & FE-1 & FE-2 & GRD & FE-1 & FE-2 & GRD \\
\hline Persistence Model & 0.9461 & 0.8982 & 0.8553 & 0.8969 & 0.8065 & 0.7934 & 0.9507 & 0.9110 & 0.8255 & 0.8727 & 0.7632 & 0.8560 \\
\hline ARIMA & 0.9272 & 0.8650 & 0.8197 & 0.8754 & 0.7660 & 0.7647 & 0.9412 & 0.8946 & 0.8016 & 0.8550 & 0.7340 & 0.8420 \\
\hline GRNN & 0.9403 & 0.8863 & 0.8475 & 0.8823 & 0.7679 & 0.7805 & 0.9324 & 0.8758 & 0.7917 & 0.8441 & 0.6930 & 0.8403 \\
\hline WNN & 0.9268 & 0.8635 & 0.8562 & 0.8716 & 0.7416 & 0.7889 & 0.9378 & 0.8727 & 0.8115 & 0.8478 & 0.6956 & 0.8398 \\
\hline ESN & 0.9460 & 0.8979 & 0.8573 & 0.8935 & 0.7921 & 0.7923 & 0.9479 & 0.9050 & 0.8217 & 0.8649 & 0.7400 & 0.8547 \\
\hline EMD-MOGWO-ESN & 0.9662 & 0.9383 & 0.8994 & 0.9382 & 0.8876 & 0.8533 & 0.9703 & 0.9483 & 0.8818 & 0.9180 & 0.8432 & 0.8999 \\
\hline VMD-MOGWO-ESN & 0.9611 & 0.9247 & 0.8880 & 0.9049 & 0.8049 & 0.8133 & 0.9587 & 0.9253 & 0.8506 & 0.8934 & 0.7810 & 0.8832 \\
\hline CEEMDAN-MOGWO-ESN & 0.9738 & 0.9501 & 0.9195 & 0.9504 & 0.9033 & 0.8835 & 0.9737 & 0.9528 & 0.8945 & 0.9410 & 0.8848 & 0.9231 \\
\hline CEEMDAN-MOPSO-ESN & 0.9515 & 0.9092 & 0.8676 & 0.9099 & 0.8284 & 0.8140 & 0.9490 & 0.9087 & 0.8236 & 0.8824 & 0.7722 & 0.8712 \\
\hline CEEMDAN-MOWCA-ESN & 0.9719 & 0.9475 & 0.9144 & 0.9412 & 0.8872 & 0.8678 & 0.9630 & 0.9352 & 0.8600 & 0.9380 & 0.8799 & 0.9209 \\
\hline
\end{tabular}

Note: The bolded values are the best values. 
Table 7. The parameters of the fitted distribution.

\begin{tabular}{ccccc}
\hline Dataset D & Gaussian & Generalized Extreme Value & Extreme Value & Logistic \\
\hline Log likelihood & -930.9960 & -942.6690 & -1170.9600 & -934.9160 \\
mean & 0.0012 & 0.0035 & -0.0329 & -0.0016 \\
variance & 0.1318 & 0.1368 & 0.2332 & 0.1380 \\
$\mu$ & 0.0012 & -0.1362 & 0.1845 & -0.0016 \\
standard error of $\boldsymbol{\mu}$ & 0.0076 & 0.0081 & 0.0083 & 0.0074 \\
$\sigma$ & 0.3630 & 0.3584 & 0.3765 & 0.2048 \\
standard error of $\sigma$ & 0.0054 & 0.0055 & 0.0055 & 0.0036 \\
$\boldsymbol{k}$ & - & -0.2272 & - & - \\
standard error of $\boldsymbol{k}$ & - & 0.0091 & - & Logistic \\
\hline Dataset E & Gaussian & Generalized Extreme Value & Extreme Value & -298.6460 \\
Log likelihood & -349.9050 & -410.3980 & -683.6500 & 0.0010 \\
mean & 0.0031 & 0.0101 & -0.0366 & 0.0774 \\
variance & 0.0794 & 0.0912 & 0.1638 & 0.0010 \\
$\boldsymbol{\mu}$ & 0.0031 & -0.1078 & 0.1455 & 0.0055 \\
standard error of $\boldsymbol{\mu}$ & 0.0059 & 0.0063 & 0.0070 & 0.1533 \\
$\sigma$ & 0.2819 & 0.2864 & 0.3155 & - \\
standard error of $\sigma$ & 0.0042 & 0.0041 & 0.0043 & - \\
$\boldsymbol{k}$ & - & -0.1963 & - & - \\
standard error of $\boldsymbol{k}$ & - & 0.0052 & - & \\
\hline Note & m & & &
\end{tabular}

Note: $\mathrm{mu}$ is the location parameter, sigma is the scale parameter, and $\mathrm{k}$ is the shape parameter.

Table 8. The interval prediction results.

\begin{tabular}{ccccccccccc}
\hline \multicolumn{9}{c}{ Dataset D } & \multicolumn{5}{c}{ Dataset E } \\
\hline Logsitc & AW & CP & AWD & CWC & SCORE & AW & CP & AWD & CWC & SCORE \\
\hline $95 \%$ & 0.2476 & 0.9930 & 0.0005 & 0.2476 & 0.2599 & 0.1042 & 0.9877 & 0.0016 & 0.1042 & 0.1176 \\
$90 \%$ & 0.2076 & 0.9894 & 0.0014 & 0.2076 & 0.4391 & 0.0875 & 0.9789 & 0.0035 & 0.0875 & 0.1984 \\
$85 \%$ & 0.1771 & 0.9859 & 0.0026 & 0.1771 & 0.5665 & 0.0766 & 0.9525 & 0.0064 & 0.0766 & 0.2641 \\
$80 \%$ & 0.1549 & 0.9736 & 0.0044 & 0.1549 & 0.6660 & 0.0766 & 0.9225 & 0.0113 & 0.0682 & 0.3217 \\
$70 \%$ & 0.1223 & 0.9419 & 0.0109 & 0.1223 & 0.8107 & 0.0551 & 0.8715 & 0.0262 & 0.0551 & 0.4118 \\
\hline Gaussian & AW & CP & AWD & CWC & SCORE & AW & CP & AWD & CWC & SCORE \\
\hline $95 \%$ & 0.1382 & 0.9613 & 0.0070 & 0.1382 & 0.1819 & 0.1868 & 1.0000 & 0.0000 & 0.1868 & 0.1980 \\
$90 \%$ & 0.1160 & 0.9349 & 0.0132 & 0.1160 & 0.3020 & 0.1566 & 1.0000 & 0.0000 & 0.1566 & 0.3320 \\
$85 \%$ & 0.1015 & 0.9243 & 0.0200 & 0.1015 & 0.3972 & 0.1336 & 0.9947 & 0.0002 & 0.1336 & 0.4261 \\
$80 \%$ & 0.1015 & 0.9049 & 0.0276 & 0.0903 & 0.4750 & 0.1169 & 0.9912 & 0.0008 & 0.1169 & 0.4995 \\
$70 \%$ & 0.0731 & 0.8539 & 0.0479 & 0.0731 & 0.5956 & 0.0922 & 0.9842 & 0.0028 & 0.0922 & 0.5977 \\
\hline
\end{tabular}




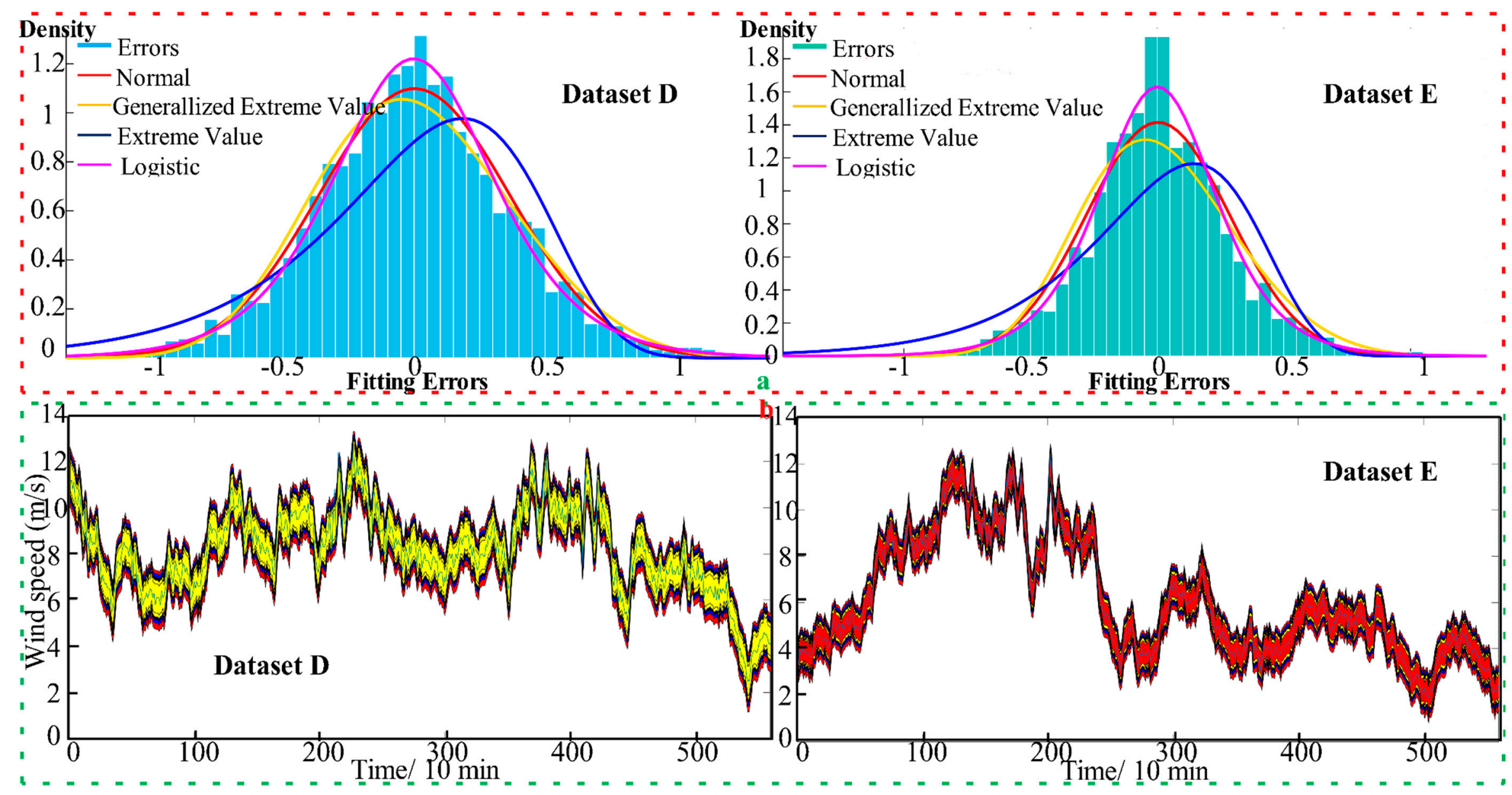

Figure 5. The distribution and interval predictions: (a) the distribution of fitting error; (b) the interval prediction results. 


\section{Discussion}

\subsection{Discussion I: Statistical Hypothesis Testing}

Though the evaluation metrics such as MAPE, AE, and RMSE can be the standard to compare the forecasting accuracy of models, statistical significance testing also need to be used to judge the models' forecasting ability [2]. Diebold-Mariano (DM) testing proposed by Diebold and Mariano in 1995 is widely used to test the significance of the forecasting ability between different methods. Based on the experiment results, this part employs DM testing to test the significance of the proposed system compared with the comparative methods to prove the superiority of the developed system in wind speed forecasting. Table 9 shows the hypothesis testing results at certain significance levels, and the results values of the experiment models are all bigger than the critical value at the $1 \%$ level or $10 \%$ level. When the results are bigger than $X_{0.01 / 2}=2.58$, it indicates the null hypothesis should be rejected at the $1 \%$ significant level, and when the results are bigger than $X_{0.1 / 2}=1.64$, it indicates the null hypothesis should be rejected at the $10 \%$ significant level. The results are all bigger than 1.64 and mostly bigger than 2.58 which means the proposed system outperforms all the comparative models at the $10 \%$ significant level, and except for CEEMDAN-MOWCA-ESN, the proposed system outperforms the comparative models at the $1 \%$ significant level. Therefore, combining the evaluation results of the experiments, we can conclude that the developed hybrid system has a better forecasting ability than the former models and can perform well in wind speed forecasting.

Table 9. Diebold-Mariano (DM) testing results.

\begin{tabular}{|c|c|c|c|c|}
\hline & A & B & $\mathrm{C}$ & D \\
\hline Persistence Model & 13.3187 * & $12.9513 *$ & $12.6671 *$ & 8.1898 * \\
\hline ARIMA & 13.0006 * & $13.4601 *$ & 12.5768 * & 9.2973 * \\
\hline GRNN & 10.5674 * & $13.7422 *$ & $10.5380 *$ & 10.0814 * \\
\hline WNN & $7.4073 *$ & $13.7422 *$ & $14.2305^{*}$ & 10.6963 * \\
\hline ESN & 13.3169 * & $13.2641 *$ & $11.9711^{*}$ & $9.1079 *$ \\
\hline EMD-MOGWO-ESN & $7.2237 *$ & $6.3326 *$ & $7.1805 *$ & $7.9541 *$ \\
\hline VMD-MOGWO-ESN & $10.0639 *$ & $10.2589 *$ & $9.9589 *$ & 7.3974 * \\
\hline CEEMDAN-MOPSO-ESN & 11.3384 * & $13.2077 *$ & $11.3404 *$ & $9.9578 *$ \\
\hline CEEMDAN-MOWCA-ESN & $1.6639^{* *}$ & $2.2310^{* *}$ & $9.2580 *$ & 2.2757 * \\
\hline \multirow[t]{2}{*}{ CEEMDAN-MOGWO-ESN } & - & - & - & - \\
\hline & $\mathbf{E}$ & $\mathbf{F}$ & G & $\mathbf{H}$ \\
\hline Persistence Model & $8.5112 *$ & $13.0287 *$ & $10.3597^{*}$ & 8.2681 * \\
\hline ARIMA & $9.1672 *$ & $13.2538 *$ & $10.6310 *$ & 8.4434 * \\
\hline GRNN & $9.5440 *$ & $13.5054^{*}$ & $10.5109 *$ & $9.2060 *$ \\
\hline WNN & $11.6328 *$ & $12.7312 *$ & $9.8560 *$ & 9.5584 * \\
\hline ESN & $8.8501 *$ & $13.2789 *$ & $10.4335^{*}$ & $8.1068 *$ \\
\hline EMD-MOGWO-ESN & $6.9389 *$ & $7.2450 *$ & $3.9875 *$ & $9.3627 *$ \\
\hline VMD-MOGWO-ESN & $6.7317 *$ & $9.9688 *$ & $7.6672 *$ & 10.3465 * \\
\hline CEEMDAN-MOPSO-ESN & $9.1159 *$ & $12.1795 *$ & $10.3353 *$ & $8.5915 *$ \\
\hline CEEMDAN-MOWCA-ESN & $3.1295 *$ & $6.5649 *$ & $8.4737 *$ & $2.6876^{*}$ \\
\hline CEEMDAN-MOGWO-ESN & - & - & - & - \\
\hline
\end{tabular}

${ }^{*}$ is at the $1 \%$ significance level; ${ }^{* *}$ is at the $10 \%$ significance level.

\subsection{Discussion II: Forecasting Stability}

An effective model can not only forecast accurately but also improve the forecasting precision stably. The forecasting accuracy has been confirmed, so this discussion is applied to demonstrate the stability of the proposed hybrid system. In general, researchers use performance variance to judge the model's stability [56]; this paper employs the forecasting error's variance to measure the stability of the developed system, and the results are placed at Table 10. As the smaller variance indicates a stronger stability, and the proposed system has the smallest variance values in all cases, we can draw a 
conclusion that the developed system owns the strongest stability compared with other methods in this research.

\subsection{Discussion III: Sensitivity Analysis of Optimization Algorithm}

There are three important parameters of the optimization algorithm MOGWO: the grey wolf number, the iteration number, and the archive size, which can significantly influence the effectiveness of the proposed hybrid system. An appropriate parameter will promote forecasting accuracy as well as forecasting efficiency, and vice versa. To explore the influence of the parameter changing to wind speed forecasting performance, we conduct this discussion by changing one of the three parameters separately and keeping the other parameters unchanged. The parameters' settings and experiment results of the discussion are shown in Table 11. From Table 11, we can find the following:

(1) The grey wolf number obviously affects the forecasting accuracy; for example, the MAPE value shows regular change with the increasing of the grey wolf number. First, the MAPE value decreases, and when the grey wolf number is set to 200 the MAPE attains a minimum; after that, the MAPE value becomes bigger again. Furthermore, with the increasing of the grey wolf number, the run time increases. Therefore, the change of the grey wolf number affects the forecasting results of the proposed system.

(2) With the changing of iteration number, the forecasting accuracy has a slight variation but the run time changes a lot. The MAPE value also decreases first and then increases, and the least MAPE is corresponding to the iteration at 50 , which is the best iteration number. Therefore, the forecasting system will slightly change with the changing of the iteration number.

(3) The forecasting accuracy of the proposed system changes better first, with the increasing of the archive size, and then grows down. It clearly revealed the archive size's effect on the performance of the proposed system.

Remark: The forecasting performance can be visibly influenced by the changing of the MOGWO's parameters. The appropriate parameters will lead to excellent forecasting performance while poor parameters cannot help to find the best initial parameters of ESN so it decreases the forecasting ability. Furthermore, the parameter settings change with the practical case conditions; the discussion proves that the grey wolf number is set as 200, the iterations are set as 50, and the archive size sets as 100 in this research.

\subsection{Discussion IV: Further Application for Multistep Forecasting Performance}

The proposed system's superiority has been completely attested in the one-step wind speed forecasting. However, the developed system only performing well in the one-step forecasting is not enough to monitor and ensure the wind power system's safety. To satisfy this requirement, multistep forecasting is conducted on the basis of dataset A and dataset C. In this experiment, we conduct a comparison between the proposed system and several traditional single methods such as the persistence model, ARIMA, neural networks, as well as several hybrid models such as EMD-MOGWO-ESN and CEEMDAN-MOWCA-ESN; the results are shown at Table 12. According to the experiment results, the developed system performs best not only in the first step but also in the second and third step, and its superiority is confirmed. Obviously, the MAPE value of the developed system is far less than the single methods and also smaller than the comparative hybrid models at each step. That means the developed system outperforms other models at multistep wind speed forecasting so it can be applied in wind energy systems. 
Table 10. Forecasting stability results.

\begin{tabular}{ccccccccc}
\hline & A & B & C & D & E & F & G & H \\
\hline Persistence Model & 0.8260 & 0.2381 & 0.1908 & 0.3723 & 0.4135 & 0.9099 & 0.1687 & 1.0664 \\
ARIMA & 0.7974 & 0.3550 & 0.2424 & 0.6507 & 0.7197 & 1.2798 & 0.2289 & 1.3046 \\
GRNN & 1.8567 & 0.2918 & 0.2112 & 0.3927 & 0.4401 & 1.0054 & 0.2475 & 1.2462 \\
WNN & 2.7276 & 0.2918 & 0.2069 & 0.3605 & 0.4163 & 1.0053 & 0.2034 & 1.2252 \\
ESN & 0.8359 & 0.2341 & 0.1869 & 0.3526 & 0.3875 & 0.8743 & 0.1708 & 1.0588 \\
EMD-MOGWO-ESN & 0.2619 & 0.0792 & 0.0607 & 0.1374 & 0.1545 & 0.3265 & 0.0566 & 0.3787 \\
VMD-MOGWO-ESN & 0.4781 & 0.1274 & 0.1298 & 0.2165 & 0.2094 & 0.6238 & 0.1032 & 0.5551 \\
CEEMDAN-MOGWO-ESN & $\mathbf{0 . 1 7 3 7}$ & $\mathbf{0 . 0 5 3 6}$ & $\mathbf{0 . 0 4 1 0}$ & $\mathbf{0 . 0 9 3 1}$ & $\mathbf{0 . 0 9 6 8}$ & $\mathbf{0 . 2 0 7 0}$ & $\mathbf{0 . 0 3 9 9}$ & $\mathbf{0 . 2 1 2 4}$ \\
CEEMDAN-MOPSO-ESN & 0.6254 & 0.1983 & 0.1479 & 0.2710 & 0.2997 & 0.6247 & 0.1498 & 0.7273 \\
CEEMDAN-MOWCA-ESN & 0.1895 & 0.0582 & 0.0814 & 0.1003 & 0.1070 & 0.2797 & 0.0838 & 0.2310 \\
\hline
\end{tabular}

Note: The bolded values are the best values.

Table 11. The sensitivity analysis results.

\begin{tabular}{|c|c|c|c|c|c|c|c|c|c|c|c|}
\hline & Number & $A E$ & MAPE & RMSE & MDAPE & $F B$ & $R$ & $I A$ & U1 & $U 2$ & TIME \\
\hline \multirow{5}{*}{ Grey Wolves Number } & 50 & 0.0107 & 2.6734 & 0.2027 & 0.0204 & -0.0018 & 0.9968 & 0.9984 & 0.0154 & 0.5179 & 183.2840 \\
\hline & 100 & 0.0056 & 2.6759 & 0.2146 & 0.0232 & -0.0009 & 0.9964 & 0.9982 & 0.0164 & 0.5016 & 252.7143 \\
\hline & 150 & 0.0150 & 2.6529 & 0.2118 & 0.0209 & -0.0025 & 0.9965 & 0.9982 & 0.0161 & 0.4930 & 376.3458 \\
\hline & 200 & 0.0067 & 2.6276 & 0.2001 & 0.0203 & -0.0011 & 0.9969 & 0.9984 & 0.0153 & 0.5064 & 437.2306 \\
\hline & 250 & 0.0096 & 2.6943 & 0.2087 & 0.0231 & -0.0016 & 0.9966 & 0.9983 & 0.0159 & 0.5010 & 535.9080 \\
\hline \multirow{5}{*}{ Iterations } & 30 & 0.0088 & 2.7023 & 0.2069 & 0.0216 & -0.0015 & 0.9967 & 0.9983 & 0.0158 & 0.5159 & 325.0705 \\
\hline & 40 & 0.0068 & 2.6998 & 0.2061 & 0.0217 & -0.0011 & 0.9967 & 0.9983 & 0.0157 & 0.5228 & 371.3325 \\
\hline & 50 & 0.0067 & 2.6276 & 0.2001 & 0.0203 & -0.0011 & 0.9969 & 0.9984 & 0.0153 & 0.5064 & 437.2306 \\
\hline & 60 & 0.0093 & 2.6327 & 0.2030 & 0.0215 & -0.0015 & 0.9968 & 0.9984 & 0.0155 & 0.4996 & 531.0117 \\
\hline & 70 & 0.0074 & 2.6346 & 0.2055 & 0.0208 & -0.0012 & 0.9967 & 0.9983 & 0.0157 & 0.4997 & 596.1518 \\
\hline \multirow{5}{*}{ Archive size } & 50 & 0.0120 & 2.6776 & 0.2130 & 0.0224 & -0.0020 & 0.9965 & 0.9982 & 0.0162 & 0.5099 & 455.5368 \\
\hline & 70 & 0.0138 & 2.6558 & 0.2031 & 0.0214 & -0.0023 & 0.9968 & 0.9984 & 0.0155 & 0.5036 & 420.2421 \\
\hline & 100 & 0.0067 & 2.6276 & 0.2001 & 0.0203 & -0.0011 & 0.9969 & 0.9984 & 0.0153 & 0.5064 & 437.2306 \\
\hline & 150 & 0.0081 & 2.6562 & 0.2038 & 0.0200 & -0.0013 & 0.9968 & 0.9984 & 0.0155 & 0.5209 & 418.6188 \\
\hline & 180 & 0.0083 & 2.7998 & 0.2120 & 0.0226 & -0.0014 & 0.9965 & 0.9982 & 0.0162 & 0.5426 & 422.0142 \\
\hline
\end{tabular}

Note: The bolded values are the results of the best parameters. 
Table 12. Multistep foresting results of the developed system.

\begin{tabular}{|c|c|c|c|c|c|c|c|c|c|}
\hline \multirow{2}{*}{ Dataset A } & \multicolumn{3}{|c|}{ 1-Step } & \multicolumn{3}{|c|}{ 2-Step } & \multicolumn{3}{|c|}{ 3-Step } \\
\hline & $\mathrm{AE}$ & MAPE & RMSE & $\mathrm{AE}$ & MAPE & RMSE & $\mathrm{AE}$ & MAPE & RMSE \\
\hline Persistence Model & -0.0069 & 7.0651 & 0.9094 & -0.0175 & 9.8131 & 1.2315 & -0.0263 & 11.7269 & 1.4890 \\
\hline ARIMA & -0.0295 & 6.9594 & 0.8940 & -0.0335 & 7.0746 & 0.9095 & -0.0292 & 7.0706 & 0.9130 \\
\hline GRNN & -0.5289 & 9.4339 & 1.4625 & -0.6208 & 12.0956 & 1.7010 & -0.7242 & 14.0128 & 1.9332 \\
\hline WNN & -0.6424 & 9.5246 & 1.7733 & -0.8249 & 13.0047 & 2.1967 & -0.9058 & 15.1044 & 2.4616 \\
\hline ESN & -0.1029 & 7.1156 & 0.9258 & -0.2130 & 9.9813 & 1.2675 & -0.3024 & 11.9022 & 1.5398 \\
\hline EMD-MOGWO-ESN & -0.0193 & 4.0520 & 0.5122 & -0.0522 & 6.3962 & 0.8779 & -0.0922 & 9.7565 & 1.3107 \\
\hline CEEMDAN-MOGWO-ESN & -0.0294 & 3.1490 & 0.4182 & -0.1027 & 6.0469 & 0.7778 & -0.1918 & 9.5652 & 1.2177 \\
\hline CEEMDAN-MOPSO-ESN & -0.1164 & 6.2568 & 0.8000 & -0.2299 & 9.0515 & 1.1618 & -0.3644 & 11.6857 & 1.4987 \\
\hline CEEMDAN-MOWCA-ESN & 0.0049 & 3.2530 & 0.4356 & 0.0061 & 6.3385 & 0.8377 & 0.0065 & 10.1770 & 1.3556 \\
\hline \multirow{2}{*}{ Dataset C } & \multicolumn{3}{|c|}{ 1-Step } & \multicolumn{3}{|c|}{ 2-Step } & \multicolumn{3}{|c|}{ 3-Step } \\
\hline & $\mathrm{AE}$ & MAPE & RMSE & $\mathrm{AE}$ & MAPE & RMSE & AE & MAPE & RMSE \\
\hline Persistence Model & 0.0021 & 6.5623 & 0.4371 & 0.0039 & 8.3148 & 0.5721 & 0.0058 & 10.2173 & 0.6808 \\
\hline ARIMA & 0.0293 & 7.5086 & 0.4937 & 0.0268 & 7.1851 & 0.4722 & 0.0250 & 7.2464 & 0.4741 \\
\hline GRNN & -0.0163 & 6.8788 & 0.4602 & -0.0349 & 8.7138 & 0.5976 & -0.0498 & 10.1368 & 0.6879 \\
\hline WNN & 0.1651 & 7.9610 & 0.4840 & 0.3099 & 11.9612 & 0.7114 & 0.4686 & 16.2716 & 0.9189 \\
\hline ESN & 0.0004 & 6.5827 & 0.4344 & 0.0009 & 8.3005 & 0.5700 & 0.0023 & 10.2097 & 0.6833 \\
\hline EMD-MOGWO-ESN & 0.0058 & 3.8554 & 0.2464 & 0.0151 & 5.9908 & 0.3989 & 0.0282 & 8.6473 & 0.5727 \\
\hline CEEMDAN-MOGWO-ESN & 0.0031 & 3.0618 & 0.2026 & 0.0058 & 5.8720 & 0.3860 & 0.0097 & 8.8344 & 0.5804 \\
\hline CEEMDAN-MOPSO-ESN & 0.0055 & 5.8461 & 0.3848 & 0.0159 & 8.4750 & 0.5606 & 0.0263 & 10.8027 & 0.7095 \\
\hline CEEMDAN-MOWCA-ESN & 0.0072 & 4.3007 & 0.2855 & 0.0160 & 7.3656 & 0.4815 & 0.0282 & 10.6772 & 0.6897 \\
\hline
\end{tabular}

Note: The bolded values are the best values. 


\section{Conclusions}

Recently, significant attention and persistent exploitation have been developed for wind energies as it is clean and renewable. However, the randomness and fluctuation of wind speeds make it hard to maintain the security and stability of wind power systems. Accurate and stable wind speed forecasting is of vital significance. Thus, this paper develops a novel hybrid forecasting system which can obtain high forecasting accuracy and stability as well as enhancing the development of wind energy. Firstly, we reduce the noise of the original wind speed via the data preprocessing method; the processed data are inputted into the ESN for forecasting, specifically, the parameters of ESN are optimized by a multi-objective algorithm to enhance the forecasting performance of the developed hybrid system. Then, four datasets of different seasons and four datasets with different features and time intervals gathered from a wind farm in Penglai China are applied to prove the effectiveness of the developed hybrid system. The developed forecasting system achieves the highest forecasting accuracy and stability in all cases. Moreover, compared with traditional statistical methods, single neural networks, as well as other hybrid methods, the proposed system can obtain the best forecasting performance. Finally, an interval forecasting is conducted to address the uncertainty problems existing in wind speed forecasting. Furthermore, several discussions from different aspects are supplemented to verify the advancement of the proposed hybrid system, showing the proposed system's excellent performance and further application. Synthesizing the experiments and discussion results, the proposed hybrid system is an effective and helpful novel method in wind speed forecasting. The system is designed based on the off-line prediction via historical wind speed data, that is the limitation of the research. Accordingly, employing the developed forecasting system conduct online prediction using real-time data should be made in future research.

Author Contributions: C.W. proposed the concept of this research and wrote the whole manuscript; J.W. provided overall guidance; C.W. carried on the data analysis; T.N. polished the manuscript and supported a part of the data processing.

Funding: This research was funded by the National Natural Science Foundation of China (Grant No. 71671029).

Conflicts of Interest: The authors declare no conflict of interest. 


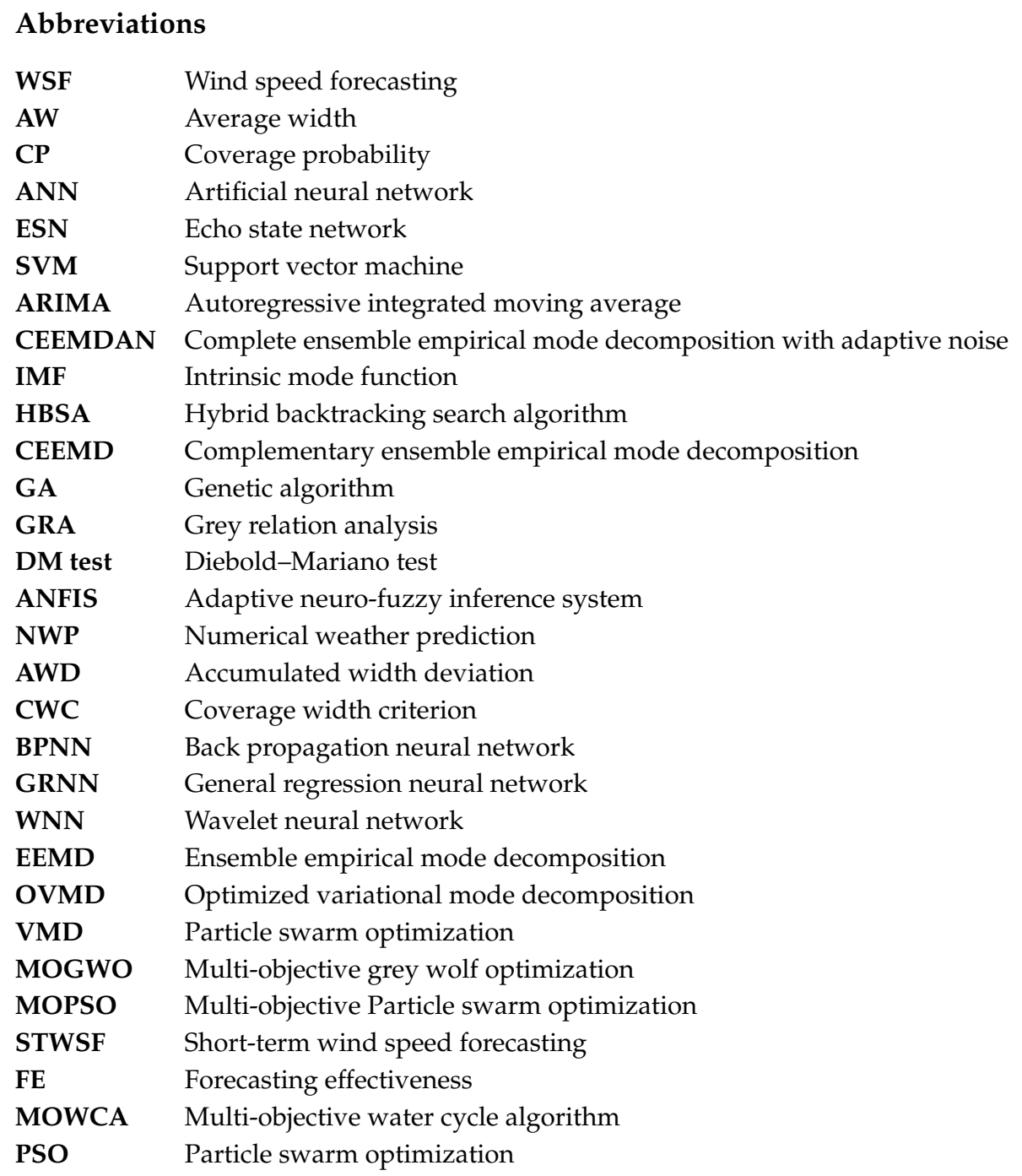

\section{References}

1. REN21. Renewables 2018 Global Status Report; REN21 Secretariatv: Paris, France, 2018; ISBN 9783981891133. Available online: http:/ / www.ren21.net (accessed on 31 December 2018).

2. Wang, J.; Du, P.; Niu, T.; Yang, W. A novel hybrid system based on a new proposed algorithm-Multi-Objective Whale Optimization Algorithm for wind speed forecasting. Appl. Energy 2017, 208, 344-360. [CrossRef]

3. Yan, J.; Liu, Y.; Han, S.; Wang, Y.; Feng, S. Reviews on uncertainty analysis of wind power forecasting. Renew. Sustain. Energy Rev. 2015, 52, 1322-1330. [CrossRef]

4. Jiang, P.; Yang, H.; Heng, J. A hybrid forecasting system based on fuzzy time series and multi-objective optimization for wind speed forecasting. Appl. Energy 2019, 235, 786-801. [CrossRef]

5. Niu, T.; Wang, J.; Lu, H.; Du, P. Uncertainty modeling for chaotic time series based on optimal multi-input multi-output architecture: Application to offshore wind speed. Energy Convers. Manag. 2018, 156, 597-617. [CrossRef]

6. Li, R.; Jin, Y. A wind speed interval prediction system based on multi-objective optimization for machine learning method. Appl. Energy 2018, 228, 2207-2220. [CrossRef]

7. Ma, X.; Jin, Y.; Dong, Q. A generalized dynamic fuzzy neural network based on singular spectrum analysis optimized by brain storm optimization for short-term wind speed forecasting. Appl. Soft Comput. J. 2017, 54, 296-312. [CrossRef]

8. Wang, J.; Yang, W.; Du, P.; Niu, T. A novel hybrid forecasting system of wind speed based on a newly developed multi-objective sine cosine algorithm. Energy Convers. Manag. 2018, 163, 134-150. [CrossRef] 
9. Zhang, W.; Qu, Z.; Zhang, K.; Mao, W.; Ma, Y.; Fan, X. A combined model based on CEEMDAN and modified flower pollination algorithm for wind speed forecasting. Energy Convers. Manag. 2017, 136, $439-451$. [CrossRef]

10. Tian, C.; Hao, Y. A Novel Nonlinear Combined Forecasting System for Short-Term Load Forecasting. Energies 2018, 11, 712. [CrossRef]

11. Becker, R.; Thrän, D. Optimal siting of wind farms in wind energy dominated power systems. Energies 2018, 11, 978. [CrossRef]

12. Ganea, D.; Amortila, V.; Mereuta, E.; Rusu, E. A joint evaluation of the wind and wave energy resources close to the Greek Islands. Sustainability 2017, 9, 1025. [CrossRef]

13. Onea, F.; Rusu, L. Evaluation of some state-of-the-art wind technologies in the nearshore of the black sea. Energies 2018, 11, 2452. [CrossRef]

14. Kollet, S.; Gasper, F.; Brdar, S.; Goergen, K.; Hendricks-Franssen, H.-J.; Keune, J.; Kurtz, W.; Küll, V.; Pappenberger, F.; Poll, S.; et al. Introduction of an Experimental Terrestrial Forecasting/Monitoring System at Regional to Continental Scales Based on the Terrestrial Systems Modeling Platform (v1.1.0). Water 2018, 10, 1697. [CrossRef]

15. Salvador, S.; Costoya, X.; Sanz-Larruga, F.J.; Gimeno, L. Development of offshore wind power: Contrasting optimal wind sites with legal restrictions in Galicia, Spain. Energies 2018, 11, 731. [CrossRef]

16. Giménez, P.O.; García-Galiano, S.G. Assessing Regional Climate Models (RCMs) Ensemble-driven reference evapotranspiration over Spain. Water 2018, 10, 1181.

17. Ganea, D.; Mereuta, E.; Rusu, L. Estimation of the Near Future Wind Power Potential in the Black Sea. Energies 2018, 11, 3198. [CrossRef]

18. Pearre, N.S.; Swan, L.G. Statistical approach for improved wind speed forecasting for wind power production. Sustain. Energy Technol. Assess. 2018, 27, 180-191. [CrossRef]

19. Hao, Y.; Tian, C. The study and application of a novel hybrid system for air quality early-warning. Appl. Soft Comput. J. 2019, 74, 729-746. [CrossRef]

20. Taghi Sattari, M.; Yurekli, K.; Pal, M. Performance evaluation of artificial neural network approaches in forecasting reservoir inflow. Appl. Math. Model. 2012, 36, 2649-2657. [CrossRef]

21. Liu, D.; Wang, J.; Wang, H. Short-term wind speed forecasting based on spectral clustering and optimised echo state networks. Renew. Energy 2015, 78, 599-608. [CrossRef]

22. Yao, X.; Wang, Z.; Zhang, H. Neurocomputing A novel photovoltaic power forecasting model based on echo state network. Neurocomputing 2019, 325, 182-189. [CrossRef]

23. Wang, J.; Yang, W.; Du, P.; Li, Y. Research and application of a hybrid forecasting framework based on multi-objective optimization for electrical power system. Energy 2018, 148, 59-78. [CrossRef]

24. Li, Y.; Wu, H.; Liu, H. Multi-step wind speed forecasting using EWT decomposition, LSTM principal computing, RELM subordinate computing and IEWT reconstruction. Energy Convers. Manag. 2018, 167, 203-219. [CrossRef]

25. Ahmed, A.; Khalid, M. An intelligent framework for short-term multi-step wind speed forecasting based on Functional Networks. Appl. Energy 2018, 225, 902-911. [CrossRef]

26. Wang, J.; Du, P.; Lu, H.; Yang, W.; Niu, T. An improved grey model optimized by multi-objective ant lion optimization algorithm for annual electricity consumption forecasting. Appl. Soft Comput. J. 2018, 72, 321-337. [CrossRef]

27. Zhao, X.; Wang, C.; Su, J.; Wang, J. Research and Application Based on the Swarm Intelligence Algorithm and Artificial Intelligence for Wind Farm Decision System. Renew. Energy 2019, 134, 681-697. [CrossRef]

28. Jaeger, H.; Haas, H. Harnessing Nonlinearity: Predicting Chaotic Systems and Saving Energy in Wireless Communication. Science 2004, 304, 78-81. [CrossRef]

29. Deihimi, A.; Orang, O.; Showkati, H. Short-term electric load and temperature forecasting using wavelet echo state networks with neural reconstruction. Energy 2013, 57, 382-401. [CrossRef]

30. Güntürkün, U. Sequential reconstruction of driving-forces from nonlinear nonstationary dynamics. Phys. D Nonlinear Phenom. 2010, 239, 1095-1107. [CrossRef]

31. Fu, T.; Wang, C. A hybrid wind speed forecasting method and wind energy resource analysis based on a swarm intelligence optimization algorithm and an artificial intelligence model. Sustainability 2018, 10, 3913. [CrossRef] 
32. Wang, Z.; Wang, C.; Wu, J. Wind energy potential assessment and forecasting research based on the data pre-processing technique and swarm intelligent optimization algorithms. Sustainability 2016, 8, 1191. [CrossRef]

33. Shi, G.; Liu, D.; Wei, Q. Neurocomputing Energy consumption prediction of of fi ce buildings based on echo state networks. Neurocomputing 2016, 216, 478-488. [CrossRef]

34. Rigamonti, M.; Baraldi, P.; Zio, E.; Roychoudhury, I.; Goebel, K.; Poll, S. Neurocomputing Ensemble of optimized echo state networks for remaining useful life prediction. Neurocomputing 2018, 281, 121-138. [CrossRef]

35. Deihimi, A.; Showkati, H. Application of echo state networks in short-term electric load forecasting. Energy 2012, 39, 327-340. [CrossRef]

36. Chouikhi, N.; Ammar, B.; Rokbani, N.; Alimi, A.M. PSO-based analysis of Echo State Network parameters for time series forecasting. Appl. Soft Comput. J. 2017, 55, 211-225. [CrossRef]

37. Wang, L.; Lv, S.; Zeng, Y. Effective sparse adaboost method with ESN and FOA for industrial electricity consumption forecasting in China. Energy 2018, 155, 1013-1031. [CrossRef]

38. Zhang, C.; Zhou, J.; Li, C.; Fu, W.; Peng, T. A compound structure of ELM based on feature selection and parameter optimization using hybrid backtracking search algorithm for wind speed forecasting. Energy Convers. Manag. 2017, 143, 360-376. [CrossRef]

39. Du, P.; Wang, J.; Guo, Z.; Yang, W. Research and application of a novel hybrid forecasting system based on multi-objective optimization for wind speed forecasting. Energy Convers. Manag. 2017, 150, 90-107. [CrossRef]

40. Naik, J.; Bisoi, R.; Dash, P.K. Prediction interval forecasting of wind speed and wind power using modes decomposition based low rank multi-kernel ridge regression. Renew. Energy 2018, 129, 357-383. [CrossRef]

41. Shrivastava, N.A.; Lohia, K.; Panigrahi, B.K. A multiobjective framework for wind speed prediction interval forecasts. Renew. Energy 2016, 87, 903-910. [CrossRef]

42. Khosravi, A.; Machado, L.; Nunes, R.O. Time-series prediction of wind speed using machine learning algorithms: A case study Osorio wind farm, Brazil. Appl. Energy 2018, 224, 550-566. [CrossRef]

43. Torres, M.E.; Colominas, M.A.; Schlotthauer, G.; Flandrin, P. A complete ensemble empirical mode decomposition with adaptive noise. In Proceedings of the 2011 IEEE International Conference on Acoustics, Speech and Signal Processing (ICASSP), Prague, Czech Republic, 22-27 May 2011; pp. 4144-4147.

44. Jaeger, H. The "echo state" approach to analysing and training recurrent neural networks-With an Erratum note 1. GMD Rep. 2010, 148, 1-47.

45. Mirjalili, S.; Saremi, S.; Mirjalili, S.M.; Coelho, L.D.S. Multi-objective grey wolf optimizer: A novel algorithm for multi-criterion optimization. Expert Syst. Appl. 2016, 47, 106-119. [CrossRef]

46. Mirjalili, S.; Mirjalili, S.M.; Lewis, A. Grey Wolf Optimizer. Adv. Eng. Softw. 2014, 69, 46-61. [CrossRef]

47. Yang, W.; Wang, J.; Niu, T.; Du, P. A hybrid forecasting system based on a dual decomposition strategy and multi-objective optimization for electricity price forecasting. Appl. Energy 2019, 235, 1205-1225. [CrossRef]

48. Jiang, P.; Ma, X. A hybrid forecasting approach applied in the electrical power system based on data preprocessing, optimization and artificial intelligence algorithms. Appl. Math. Model. 2016, 40, 10631-10649. [CrossRef]

49. Wang, J.; Wang, Y.; Jiang, P. The study and application of a novel hybrid forecasting model—A case study of wind speed forecasting in China. Appl. Energy 2015, 143, 472-488. [CrossRef]

50. Xiao, L.; Shao, W.; Wang, C.; Zhang, K.; Lu, H. Research and application of a hybrid model based on multi-objective optimization for electrical load forecasting. Appl. Energy 2016, 180, 213-233. [CrossRef]

51. Zhang, C.; Wei, H.; Xie, L.; Shen, Y.; Zhang, K. Direct interval forecasting of wind speed using radial basis function neural networks in a multi-objective optimization framework. Neurocomputing 2016, 205, 53-63. [CrossRef]

52. Wang, J.; Niu, T.; Lu, H.; Guo, Z.; Yang, W.; Du, P. An analysis-forecast system for uncertainty modeling of wind speed: A case study of large-scale wind farms. Appl. Energy 2018, 211, 492-512. [CrossRef]

53. Qin, S.; Liu, F.; Wang, J.; Song, Y. Interval forecasts of a novelty hybrid model for wind speeds. Energy Rep. 2015, 1, 8-16. [CrossRef]

54. Inman, R.H.; Pedro, H.T.C.; Coimbra, C.F.M. Solar forecasting methods for renewable energy integration. Prog. Energy Combust. Sci. 2013, 39, 535-576. [CrossRef] 
55. Wang, J.; Song, Y.; Liu, F.; Hou, R. Analysis and application of forecasting models in wind power integration: A review of multi-step-ahead wind speed forecasting models. Renew. Sustain. Energy Rev. 2016, 60, 960-981. [CrossRef]

56. Tian, C.; Hao, Y.; Hu, J. A novel wind speed forecasting system based on hybrid data preprocessing and multi-objective optimization. Appl. Energy 2018, 231, 301-319. [CrossRef] 